\title{
16- and 18-Electron Ruthenium(II) Complexes of the Neutral, Potentially Tridentate Triamine Ligand 2,6-[Bis(dimethylamino)methyl]pyridine $\left(\mathrm{NN}^{\prime} \mathrm{N}\right)$
}

\author{
Rob A. T. M. Abbenhuis,${ }^{\dagger}$ Ignacio del Río, ${ }^{\dagger}$ Michel M. Bergshoef,${ }^{\dagger}$ Jaap Boersma, ${ }^{\dagger}$ \\ Nora Veldman, ${ }^{*}$ Anthony L. Spek, ${ }^{\ddagger} \perp$ and Gerard van Koten*, ${ }^{*}$ \\ Debye Institute, Department of Metal-Mediated Synthesis, and Bijvoet Center for Biomolecular \\ Research, Laboratory of Crystal and Structural Chemistry, Utrecht University, Padualaan 8, \\ $3584 \mathrm{CH}$ Utrecht, The Netherlands
}

Received August 20, 1997

The potentially tridentate coordinating ligands $\mathbf{N N}^{\prime} \mathbf{N}$ (2,6-bis[(dimethylamino)methyl]pyridine) and PNP (2,6bis[(diphenylphosphino)methyl]pyridine) react with $\left[\mathrm{RuCl}_{2}\left(\mathrm{PPh}_{3}\right)_{3}\right]$ to give $\left[\right.$ mer,trans $\left.-\mathrm{RuCl}_{2}\left(\mathbf{N N}^{\prime} \mathbf{N}\right)\left(\mathrm{PPh}_{3}\right)\right](\mathbf{1})$ and $\left[\right.$ mer,trans $\left.-\mathrm{RuCl}_{2}(\mathbf{P N P})\left(\mathrm{PPh}_{3}\right)\right](\mathbf{2})$, respectively. Complex 1 functions as a starting material for a variety of $\mathrm{Ru}\left[\mathbf{N N}^{\prime} \mathbf{N}\right]$ complexes. It reacts with either 1 or 2 equiv of $\mathrm{AgOTf}\left(\mathrm{OTf}^{-}=\mathrm{SO}_{3} \mathrm{CF}_{3}{ }^{-}\right)$to yield monocationic $\left[\operatorname{RuCl}\left(\mathbf{N N}^{\prime} \mathbf{N}\right)\left(\mathrm{PPh}_{3}\right)\right] \mathrm{OTf}(\mathbf{3})$ and $\left[\operatorname{RuOTf}\left(\mathbf{N N}^{\prime} \mathbf{N}\right)\left(\mathrm{PPh}_{3}\right)\right] \mathrm{OTf}(\mathbf{4})$, respectively. The molecular structure of 4 shows a pentacoordinated ruthenium(II) center with a square-pyramidal environment. The reaction of $\mathbf{1}$ with either 1 or 2 equiv of AgOTf in the presence of $\mathrm{MeCN}$ results in the formation of the six-coordinate mono- or dicationic complexes $\left[\right.$ mer-RuCl$\left.(\mathrm{MeCN})\left(\mathbf{N N}^{\prime} \mathbf{N}\right)\left(\mathrm{PPh}_{3}\right)\right] \mathrm{X}(\mathbf{6 a})\left(\mathrm{X}=\mathrm{OTf}^{-}\right)$and $\left[\right.$mer,trans $\left.-\mathrm{Ru}(\mathrm{MeCN})_{2}\left(\mathbf{N N}^{\prime} \mathbf{N}\right)\left(\mathrm{PPh}_{3}\right)\right]\left(\mathrm{OTf}_{2}\right)_{2}$ (7), respectively. At $55{ }^{\circ} \mathrm{C}$ in acetonitrile, $\mathbf{1}$ converts quantitatively into $\mathbf{6 b}\left(\mathrm{X}=\mathrm{Cl}^{-}\right)$which is isostructural with 6a. Reaction of polymeric $\left[\mathrm{RuCl}_{2}(\mathrm{nbd})\right]_{n}\left(\mathrm{nbd}=2,5\right.$-norbornadiene) with $\mathbf{N} \mathbf{N}^{\prime} \mathbf{N}$ under a nitrogen atmosphere leads to the formation of neutral, dinitrogen-bridged dimeric $\left[\left(\mu-\mathrm{N}_{2}\right)\left(\text { mer,trans }-\mathrm{RuCl}_{2}\left\{\mathbf{N N}^{\prime} \mathbf{N}\right\}\right)_{2}\right](\mathbf{8})$. The dinitrogen ligand is readily replaced when $\mathbf{8}$ is treated with $\mathrm{CO}$, giving the neutral complex $\left[\right.$ mer,trans $\left.-\mathrm{RuCl}_{2}(\mathrm{CO})\left(\mathbf{N N}^{\prime} \mathbf{N}\right)\right]$ (9). The 16-electron complex 3 reacts with $\mathrm{CO}$ giving $\left[\right.$ mer, cis- $\left.\mathrm{RuCl}(\mathrm{CO})_{2}\left(\mathbf{N N}^{\prime} \mathbf{N}\right)\right] \mathrm{OTf}(\mathbf{1 0})$, involving the formation of $\left[\mathrm{RuCl}(\mathrm{CO})\left(\mathbf{N N}^{\prime} \mathbf{N}\right)\left(\mathrm{PPh}_{3}\right)\right] \mathrm{OTf}(\mathbf{1 1})$, followed by substitution of $\mathrm{PPh}_{3}$ by a second CO molecule. This stepwise mechanism was confirmed by the isolation of 11. The 16-electron complex 4 reacts with $\mathrm{CO}$ under the formation of monocationic $\left[\right.$ mer-RuOTf $\left.(\mathrm{CO})\left(\mathbf{N N}^{\prime} \mathbf{N}\right)\left(\mathrm{PPh}_{3}\right)\right] \mathrm{OTf}(\mathbf{1 2})$ and a number of unidentified complexes. In the presence of ethyl alcohol, 4 reacts with $\mathrm{CO}$ to give monocationic $\left[\right.$ mer $-\operatorname{RuOTf}(\mathrm{CO})\left(\mathbf{N N}^{\prime} \mathbf{N}\right)-$ (HOEt)]OTf (13). The X-ray crystal structures of $\mathbf{1 , ~ 4 , ~ 6 a , ~ 8 , ~ 1 1 , ~ a n d ~} \mathbf{1 3}$ are reported in abbreviated form.

\section{Introduction}

Although a wide range of (chiral) bidentate ligands has been used in (asymmetric) catalysis, ${ }^{1-3}$ application of terdentate, especially neutral $C_{2}$ symmetrical, ligands in homogeneous catalysis $^{3-4}$ has received much less attention. Recently, a number of such terdentate ligands, bearing three $\mathrm{sp}^{2}$-hybridized nitrogen donor atoms, have been reported, e.g. bis(oxazolinyl)pyridines 5 and bis(pyrazolyl)pyridines ("pybox"). 6 Moreover, a number of related $C_{2}$ symmetrical, neutral, mixed phosphorusnitrogen tridentate ligands of type PNP have been reported, in

* To whom correspondence should be addressed. E-mail address: g.vankoten@chem.ruu.nl. Fax: +31 302523615.

Debye Institute.

$\doteqdot$ Bijvoet Center for Biomolecular Research.

$\perp$ To whom correspondence on crystallographic studies should be addressed. E-mail address: spea@chem.ruu.nl.

(1) Togni, A.; Venanzi, L. M. Angew. Chem., Int. Ed. Engl. 1994, 33, 497-526.

(2) Noyori, R. Asymmetric Catalysis in Organic Synthesis; Wiley: New York, 1994.

(3) Taqui Khan, M. M.; Martell, A. E. Homogeneous Catalysis by Metal Complexes; Academic Press: New York, 1974; Vols. I and II.

(4) (a) For a review on multidentate phosphines, see: Mayer, H. A.; Kaska, W. C. Chem. Rev . 1994, 94, 139. (b) For a review on the $\mathbf{N C N}^{-}$ligand, see: Rietveld, M.; Grove, D. M.; van Koten, G. New J. Chem. 1997, $21,751-771$.

(5) (a) Nishiyama, H.; Sakaguki, H.; Nakamura, T.; Horihata, M.; Kondo, M.; Itoh, K. Organometallics 1989, 9, 846. (b) Nishiyama, H.; Kondo, M.; Nakamura, T.; Hoh, K. Organometallics 1991, 10, 500.

(6) Christenson, D. L.; Toker, C. J.; Tolman, W. B. Organometallics 1995, 14, 2148. which a central pyridine ring contains bis-ortho (a)chiral phosphine substituents. ${ }^{7}$ We are currently investigating the use of monoanionic, terdentate ligand systems $\left[\mathrm{C}_{6} \mathrm{H}_{3}\left(\mathrm{CH}_{2} \mathrm{NMe}_{2}\right)_{2}\right.$ $2,6]^{-}\left([\mathbf{N C N}]^{-}\right)$or $\left[\mathrm{C}_{6} \mathrm{H}_{3}\left(\mathrm{CH}_{2} \mathrm{PPh}_{2}\right)_{2}-2,6\right]^{-}\left([\mathbf{P C P}]^{-}\right)$which differ from neutral tridentate ligands $\mathbf{N N}^{\prime} \mathbf{N}$ and PNP by the fact that NCN and PCP are bonded to the (transition) metal by a metal-carbon $\sigma$ bond (see Figure 1). ${ }^{8,9}$

Ruthenium(II) complexes containing the $[\mathbf{N C N}]^{-}$and $[\mathbf{P C P}]^{-}$ ligand systems have proven to be very active catalysts in base co-catalyzed hydrogen-transfer reactions of ketones and imines with 2-propanol. ${ }^{10,11}$ In these reactions, turnover numbers up to 25000 were observed. ${ }^{12}$ Recently, we have also developed $C_{2}$ symmetric chiral analogs of the $[\mathbf{N C N}]^{-}$ligand by introducing chirality at the benzylic carbon atom positions. ${ }^{13}$ In order to study the effect of the replacement of a metal-carbon $\sigma$ bond in NCN and PCP metal complexes by a metal-nitrogen

(7) (a) Jiang, Q.; Van Plew, D.; Murtuza, S.; Zhang, X. Tetrahedron Lett. 1996, 37, 797. (b) Sablong, R.; Newton, C.; Dierkes, P.; Osborn, J. A. Tetrahedron Lett. 1996, 37, 4933. (c) Sablong, R.; Osborn, J. A. Tetrahedron Lett. 1996, 37, 4937. (d) Barloy, L.; Ku, S. Y.; Osborn, J. A.; De Cian, A.; Fischer, J. Polyhedron 1997, 16, 291.

(8) van Koten, G. Pure Appl. Chem. 1989, 61, 1681-1694.

(9) Karlen, T.; van Koten, G. Manuscript in preparation.

(10) Sutter, J.-P.; Steenwinkel, P.; Karlen, T.; Grove, D. M.; Veldman, N.; Smeets, W. J. J.; Spek, A. L.; van Koten, G. Organometallics 1996, $15,941-948$.

(11) Karlen, T.; Dani, P.; Grove, D. M.; Steenwinkel, P.; van Koten, G. Organometallics 1996, 15, 5687-5694.

(12) Karlen, T.; Grove, D. M.; van Koten, G. Manuscript in preparation. 
<smiles>Cc1c(CN(C)C)cccc1CN(C)C</smiles>

$[\mathrm{NCN}]^{-}$

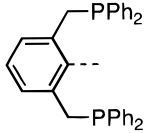

[PCP]
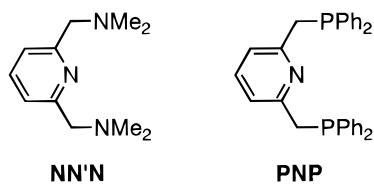

Figure 1. Ligands used and their abbreviations.

coordinate bond, we applied the neutral pyridine analogue $\left[\mathrm{C}_{5} \mathrm{H}_{3} \mathrm{~N}\left(\mathrm{CH}_{2} \mathrm{NMe}_{2}\right)_{2}-2,6\right]$ ( $\left.\mathbf{N N}^{\prime} \mathbf{N}\right)$ to ruthenium coordination chemistry. ${ }^{14}$ Like the $[\mathbf{N C N}]^{-}$ligand, neutral $\mathbf{N N}^{\prime} \mathbf{N}$ contains two flexible ortho- $\mathrm{CH}_{2} \mathrm{NMe}_{2}$ substituents, each carrying a tertiary amine donor atom. Furthermore, the $\mathbf{N N}^{\prime} \mathbf{N}$ ligand has some similarity with the well-known $2,2^{\prime}, 6: 2^{\prime \prime}$-terpyridine (terpy) but differs with respect to the nature of two of the N-donor atoms. Where terpyridine contains three $\mathrm{sp}^{2}$-hybridized nitrogen donor atoms, the $\mathbf{N} \mathbf{N}^{\prime} \mathbf{N}$ ligand is based on a combination of one $\mathrm{sp}^{2}$ - and two $\mathrm{sp}^{3}$-hybridized donor atoms. Moreover, the use of terpyridine is restricted to 18-electron complexes. In this paper, we report on the synthesis, characterization, and reactivity of 16- and 18-electron ruthenium(II) complexes of the $\mathbf{N N}^{\prime} \mathbf{N}$ ligand $\left[\mathrm{C}_{5} \mathrm{H}_{3} \mathrm{~N}\left(\mathrm{CH}_{2} \mathrm{NMe}_{2}\right)_{2}-2,6\right]$.

\section{Experimental Section}

Syntheses were performed in a dry, oxygen-free nitrogen atmosphere using standard Schlenk techniques. $\mathrm{Et}_{2} \mathrm{O}$ (predried on $\mathrm{CaH}_{2}$ ) and pentane were carefully dried and distilled from sodium benzophenone ketyl prior to use. ${ }^{1} \mathrm{H},{ }^{13} \mathrm{C}$, and, ${ }^{31} \mathrm{P}$ NMR spectra were recorded on 200 and $300 \mathrm{MHz}$ spectrometers at $298 \mathrm{~K} .{ }^{1} \mathrm{H}$ and ${ }^{13} \mathrm{C}$ chemical shifts are referenced to $\mathrm{Me}_{4} \mathrm{Si}$, and ${ }^{31} \mathrm{P} \mathrm{NMR}$ data to $85 \% \mathrm{H}_{3} \mathrm{PO}_{4}$, respectively. ${ }^{19} \mathrm{~F}$ shifts were referenced to $\mathrm{CFCl}_{3}$. IR measurements were made on a FT-IR spectrophotometer set to a resolution of $2 \mathrm{~cm}^{-1}$. Raman measurements were performed on a spectrophotometer equipped with a Nd:YAG laser using a laser power of $10 \mathrm{~mW}$ set to a resolution of $2 \mathrm{~cm}^{-1}$ in combination with an InGaAs detector. FAS-MS spectra were recorded using $\mathrm{Cs}$ as a bombardment gas and NBA as a matrix. Elemental analyses were carried out by Dornis und Kölbe, Microanalytisches Laboratorium (Mülheim a.d. Ruhr, Germany). $\mathrm{RuCl}_{2}\left(\mathrm{PPh}_{3}\right)_{3},{ }^{15}$ $\mathrm{RuCl}_{2}\left(\mathrm{PMe}_{3}\right)_{4},{ }^{16}\left[\mathrm{RuCl}_{2} \text { (norbornadiene) }\right]_{n},{ }^{17}$ 2,6-bis[(dimethylamino)methyl]pyridine $\left(\mathbf{N N}^{\prime} \mathbf{N}\right),{ }^{14}$ and 2,6-bis[(diphenylphosphino)methyl]pyridine (PNP) ${ }^{18}$ were prepared according to literature procedures. Full ${ }^{1} \mathrm{H},{ }^{13} \mathrm{C}$, and ${ }^{31} \mathrm{P}$ NMR data of the ligands are added as Supporting Information.

Synthesis of [mer,trans- $\left.\mathbf{R u C l}_{2}\left(\mathbf{N N}^{\prime} \mathbf{N}\right)\left(\mathbf{P P h}_{3}\right)\right]$ (1). To a solution of $\mathrm{RuCl}_{2}\left(\mathrm{PPh}_{3}\right)_{3}(0.67 \mathrm{~g}, 0.70 \mathrm{mmol})$ in $\mathrm{CH}_{2} \mathrm{Cl}_{2}(50 \mathrm{~mL})$ was added a solution of $\mathbf{N N}^{\prime} \mathbf{N}(0.14 \mathrm{~g}, 0.70 \mathrm{mmol})$ in $\mathrm{CH}_{2} \mathrm{Cl}_{2}(5 \mathrm{~mL})$ over $1 \mathrm{~min}$. After the mixture was stirred for $30 \mathrm{~min}$, the solvent was removed in vacuo. The remaining brown oil was extracted with $\mathrm{Et}_{2} \mathrm{O}(10 \mathrm{~mL}$, $2 \times)$ leaving a yellow brown solid as the pure product $(0.42 \mathrm{~g}, 96 \%)$. No decomposition at $T<200{ }^{\circ} \mathrm{C}$. ${ }^{1} \mathrm{H}$ NMR $\left(\mathrm{CDCl}_{3}, \mathrm{ppm}\right): \delta 8.15$ (t, $\left.6 \mathrm{H}, o-\mathrm{H} \mathrm{PPh}_{3}\right), 7.65$ (t, $1 \mathrm{H}, p-\mathrm{H}$ py), 7.34-7.25 (m, $11 \mathrm{H}, m-\mathrm{H}$ py, ar- $\mathrm{H}), 4.00\left(\mathrm{~s}, 4 \mathrm{H}, \mathrm{CH}_{2}\right), 2.10\left(\mathrm{~s}, 12 \mathrm{H}, \mathrm{NMe}_{2}\right) .{ }^{13} \mathrm{C} \mathrm{NMR}\left(\mathrm{CDCl}_{3}\right.$, ppm): $\delta 161.7\left(o-\mathrm{C}\right.$ py), $138.9\left(\mathrm{~d},{ }^{1} J_{\mathrm{PC}}=31.7 \mathrm{~Hz}\right.$, ipso-C PPh), 135.0 ( $p$-C py), $134.4\left(\mathrm{~d},{ }^{3} J_{\mathrm{PC}}=9.2 \mathrm{~Hz}, m-\mathrm{C} \mathrm{PPh}_{3}\right), 128.3(p-\mathrm{C} \mathrm{PPh}), 127.5$ $\left(\mathrm{d},{ }^{2} J_{\mathrm{PC}}=8.3 \mathrm{~Hz}, o-\mathrm{C} \mathrm{PPh}_{3}\right), 119.3\left(m-\mathrm{C}\right.$ py). ${ }^{31} \mathrm{P} \mathrm{NMR}\left(\mathrm{CDCl}_{3}\right.$, ppm): $\delta 37.0\left(\mathrm{PPh}_{3}\right) . \mathrm{UV} / \mathrm{vis}, \lambda_{\max }(\epsilon): 390$ (4949). Anal. Calcd for $\mathrm{C}_{29} \mathrm{H}_{34} \mathrm{Cl}_{2} \mathrm{~N}_{3} \mathrm{PRu}$ : C, 55.50; H, 5.46; N, 6.70. Found: C, 55.38; $\mathrm{H}$, 5.37; N, 6.66.

Synthesis of $\left[m e r\right.$, trans $\left.-\mathbf{R u C l}_{2}(\mathbf{P N P})\left(\mathbf{P P h}_{3}\right)\right](\mathbf{2}){ }^{7 \mathrm{~d}}$ To a solution of $\mathrm{RuCl}_{2}\left(\mathrm{PPh}_{3}\right)_{3}(3.76 \mathrm{~g}, 3.92 \mathrm{mmol})$ in $\mathrm{CH}_{2} \mathrm{Cl}_{2}(50 \mathrm{~mL})$ was added a

(13) Donkervoort, J. G.; Jastrzebski, J. T. B. H.; van Koten, G. J. Organomet. Chem., in press.

(14) Markies, B. M.; Wijkens, P.; Kooijman, H.; Veldman, N.; Spek, A. L.; Boersma, J.; van Koten, G. Organometallics 1994, 13, 32443258 .

(15) Holm, R. Inorg. Synth. 1970, 12, 238.

(16) Singer, H. E.; Hademer, E.; Oechmichen, U.; Dixneuf, P. J. Organomet. Chem. 1979, 178, C13.

(17) Abel, E. W.; Bennett, M. A.; Wilkinson, G. J. Chem. Soc. 1959, 3178.

(18) Danlhoff, W. V.; Nelson, S. M. J. Chem. Soc. A 1971, 2184. solution of PNP $(1.86 \mathrm{~g}, 3.92 \mathrm{mmol})$ in $\mathrm{CH}_{2} \mathrm{Cl}_{2}(10 \mathrm{~mL})$. After the mixture was stirred for $30 \mathrm{~min}$, the solvent was removed in vacuo and the residue extracted with $\mathrm{Et}_{2} \mathrm{O}(20 \mathrm{~mL}, 2 \times)$, leaving the pure product as a yellow solid $(3.35 \mathrm{~g}, 94 \%)$. No decomposition at $T<200{ }^{\circ} \mathrm{C} .{ }^{1} \mathrm{H}$ NMR $\left(\mathrm{CDCl}_{3}, \mathrm{ppm}\right): \delta 7.53(\mathrm{t}, 1 \mathrm{H}, p-\mathrm{H}$ py), $7.45-7.20(\mathrm{~m}, 22 \mathrm{H}$, ar$\mathrm{H}), 7.15-7.00(\mathrm{~m}, 10 \mathrm{H}$, ar- $\mathrm{H}), 6.90-6.85(\mathrm{~m}, 5 \mathrm{H}, m-\mathrm{H}$ py and ar- $\mathrm{H})$, $4.55\left(\mathrm{t},\left|{ }^{2} J_{\mathrm{HP}}+{ }^{4} J_{\mathrm{HP}}\right|=9.4 \mathrm{~Hz}, 4 \mathrm{H}, \mathrm{CH}_{2}\right) .{ }^{13} \mathrm{C} \mathrm{NMR}\left(\mathrm{CDCl}_{3}, \mathrm{ppm}\right)$ : $\delta 162.36\left(o-\mathrm{C}\right.$ pyr), $137.75\left(\mathrm{~d},{ }^{1} J_{\mathrm{PC}}=39.9 \mathrm{~Hz}\right.$, ipso-C $\left.\mathrm{PPh}_{2}\right), 136.55$ $\left(p-\mathrm{C}\right.$ py), $134.82\left(\mathrm{~d},{ }^{3} J_{\mathrm{PC}}=9.3 \mathrm{~Hz}, m-\mathrm{C} \mathrm{PPh}_{3}\right), 133.78\left(\mathrm{t}, J_{\mathrm{PC}}=9.6\right.$ $\left.\mathrm{Hz}, m-\mathrm{C} \mathrm{PPh}{ }_{2}\right), 133.12$ (t, $J_{\mathrm{PC}}=38.0 \mathrm{~Hz}$, ipso-C $\left.\mathrm{PPh}_{3}\right), 129.03(p-\mathrm{C}$ $\left.\mathrm{PPh}_{2}\right), 128.36\left(p-\mathrm{C} \mathrm{PPh}_{3}\right), 127.54\left(\mathrm{t}, J_{\mathrm{PC}}=8.4 \mathrm{~Hz}, o-\mathrm{C} \mathrm{PPh}_{2}\right), 127.74$ $\left(\mathrm{d}, J_{\mathrm{PC}}=8.9 \mathrm{~Hz}, o-\mathrm{C} \mathrm{PPh}\right)_{3}, 120.47(m-\mathrm{C} \mathrm{py}), 46.98\left(\mathrm{t}, J_{\mathrm{PC}}=4.1 \mathrm{~Hz}\right.$, $\left.\mathrm{CH}_{2}\right)$. ${ }^{31} \mathrm{P}$ NMR $\left(\mathrm{CDCl}_{3}, \mathrm{ppm}\right): \delta 41.83\left(\mathrm{t}, 1 \mathrm{P}, J_{\mathrm{PP}}=29 \mathrm{~Hz}, \mathrm{PPh}_{3}\right)$, $35.41\left(\mathrm{~d}, 2 \mathrm{P}, J_{\mathrm{PP}}=29 \mathrm{~Hz}, \mathrm{PPh}_{2}\right)$. UV/vis, $\lambda_{\max }(\epsilon): 327$ (5136), 398 (2351). Anal. Calcd for $\mathrm{C}_{49} \mathrm{H}_{42} \mathrm{Cl}_{2} \mathrm{~N}_{3} \mathrm{P}_{3} \mathrm{Ru}$ : C, 64.69; H, 4.65; N, 1.54. Found: $\mathrm{C}, 64.55 ; \mathrm{H}, 4.40 ; \mathrm{N}, 1.47$.

Synthesis of $\left[\mathbf{R u C l}\left(\mathbf{N N}^{\prime} \mathbf{N}\right)\left(\mathbf{P P h}_{3}\right)\right] \mathbf{O T f}(\mathbf{3})$. To a solution of $\mathbf{1}(0.65$ $\mathrm{g}, 1.04 \mathrm{mmol})$ in $\mathrm{CH}_{2} \mathrm{Cl}_{2}(50 \mathrm{mmol})$ was added solid AgOTf $(0.27 \mathrm{~g}$, $1.04 \mathrm{mmol})$. After it was stirred for $45 \mathrm{~min}$, the reaction mixture had changed color from brown to purple, and a newly formed flaky white precipitate was filtered off. The volume of the remaining solution was reduced in vacuo to $3 \mathrm{~mL}$. Pentane $(20 \mathrm{~mL})$ was added upon which the product precipitated. After filtration and subsequent drying in vacuo, the pure product was isolated as a purple solid $(0.61 \mathrm{~g}, 92 \%)$. No decomposition at $T<200{ }^{\circ} \mathrm{C}$. ${ }^{1} \mathrm{H}$ NMR (acetone- $\left.d_{6}, \mathrm{ppm}\right)$ : $\delta$ 7.90-7.80 (m, 6H, m-H PPh $), 7.48-7.30(\mathrm{~m}, 12 \mathrm{H}$, ar-H and py-H), $4.05\left(\mathrm{~d},{ }^{2} J_{\mathrm{HAHB}}=16.2 \mathrm{~Hz}, \mathrm{CH}_{A} \mathrm{H}_{\mathrm{B}}\right), 3.51\left(\mathrm{~d},{ }^{2} J_{\mathrm{HAHB}}=16.2 \mathrm{~Hz}, \mathrm{CH}_{\mathrm{A}} H_{B}\right)$, $2.68(\mathrm{~s}, 6 \mathrm{H}, \mathrm{N}(\mathrm{Me})(\mathrm{Me})), 2.32(\mathrm{~s}, 6 \mathrm{H}, \mathrm{N}(\mathrm{Me})(\mathrm{Me})) .{ }^{13} \mathrm{C} \mathrm{NMR}$ (acetone$\left.d_{6}, \mathrm{ppm}\right): \delta 162.7$ (o-C py), 137.3 ( $p$-C py), $134.4\left(\mathrm{~d},{ }^{3} J_{\mathrm{PC}}=9.9 \mathrm{~Hz}\right.$, $m$-C $\left.\mathrm{PPh}_{3}\right), 134.3\left(\mathrm{~d},{ }^{1} J_{\mathrm{PC}}=51.9 \mathrm{~Hz}\right.$, ipso-C $\left.\mathrm{PPh}_{3}\right), 131.2\left(\mathrm{~d},{ }^{4} J_{\mathrm{PC}}=\right.$ $\left.2.7 \mathrm{~Hz}, p-\mathrm{C} \mathrm{PPh}_{3}\right), 129.2\left(\mathrm{~d},{ }^{2} J_{\mathrm{PC}}=10.5 \mathrm{~Hz}, o-\mathrm{C} \mathrm{PPh}\right)_{3}, 122.6(m-\mathrm{C}$ py), $73.7\left(\mathrm{CH}_{2}\right), 52.8(\mathrm{~N}(\mathrm{Me})(\mathrm{Me})), 47.6(\mathrm{~N}(\mathrm{Me})(\mathrm{Me})) .{ }^{31} \mathrm{P}$ NMR (acetone- $\left.d_{6}, \mathrm{ppm}\right): \delta 86.6\left(\mathrm{PPh}_{3}\right) . \mathrm{UV} / \mathrm{vis}, \lambda_{\max }(\epsilon): 365(11144), 530$ (3500). Anal. Calcd for $\mathrm{C}_{30} \mathrm{H}_{34} \mathrm{ClF}_{3} \mathrm{~N}_{3} \mathrm{O}_{3} \mathrm{PRuS}$ : C, 48.61; $\mathrm{H}, 4.62$; N, 5.67. Found: C, $48.25 ; \mathrm{H}, 4.44, \mathrm{~N}, 5.66$.

Synthesis of [RuOTf(NN'N)(PPh $\left.\mathbf{P h}_{3}\right)$ OTf (4). To a solution of $\mathbf{1}$ (1 $\mathrm{g}, 1.6 \mathrm{mmol})$ in $\mathrm{CH}_{2} \mathrm{Cl}_{2}(50 \mathrm{~mL})$ was added $\operatorname{AgOTf}(0.85 \mathrm{~g}, 3.2 \mathrm{mmol})$. After the suspension was stirred for $30 \mathrm{~min}$, a newly formed flaky white precipitate was filtered off, and from the remaining purple solution the solvent was removed in vacuo, leaving a purple solid as the pure product $(1.27 \mathrm{~g}, 93 \%)$. No decomposition at $T<200{ }^{\circ} \mathrm{C}$. ${ }^{1} \mathrm{H}$ NMR $\left(\mathrm{CDCl}_{3}, \mathrm{ppm}\right): \delta 7.75$ (t, $1 \mathrm{H}, p-\mathrm{H}$ py), 7.49-7.40 (m, 9H, ar-H), 7.32$7.25(\mathrm{~m}, 8 \mathrm{H}, \mathrm{ar}-\mathrm{H}), 3.95\left(\mathrm{~d},{ }^{2} J_{\mathrm{HAHB}}=16 \mathrm{~Hz}, \mathrm{CH}_{A} \mathrm{H}_{\mathrm{B}}\right), 3.21\left(\mathrm{~d},{ }^{2} J_{\mathrm{HAHB}}\right.$ $\left.=16 \mathrm{~Hz}, \mathrm{CH}_{\mathrm{A}} H_{B}\right), 2.71(\mathrm{~s}, 6 \mathrm{H}, \mathrm{N}(\mathrm{Me})(\mathrm{Me})), 2.31(\mathrm{~s}, 6 \mathrm{H},(\mathrm{NMe})(M e))$. ${ }^{13} \mathrm{C}$ NMR $\left(\mathrm{CDCl}_{3}, \mathrm{ppm}\right): \delta 162.6(o-\mathrm{C}$ py), $137.8(p-\mathrm{C}$ py), $133.4(\mathrm{~d}$, $\left.{ }^{3} J_{\mathrm{PC}}=9.6 \mathrm{~Hz}, m-\mathrm{C} \mathrm{PPh}_{3}\right), 131.6\left(\mathrm{~d},{ }^{1} J_{\mathrm{PC}}=46.1 \mathrm{~Hz}\right.$, ipso-C $\left.\mathrm{PPh}_{3}\right)$, $131.3(p-\mathrm{C} \mathrm{PPh}), 128.9\left({ }^{2} J_{\mathrm{PC}}=10.6 \mathrm{~Hz}, o-\mathrm{C} \mathrm{PPh}{ }_{3}\right), 122.6(m-\mathrm{C}$ py), $118.50\left(\mathrm{q},{ }^{1} J_{\mathrm{CF}}=310 \mathrm{~Hz}, \mathrm{SO}_{3} \mathrm{CF}_{3}\right), 72.1\left(\mathrm{CH}_{2}\right), 52.6(\mathrm{~N}(\mathrm{Me})(\mathrm{Me}))$, $45.7(\mathrm{~N}(\mathrm{Me})(\mathrm{Me})) .{ }^{31} \mathrm{P} \mathrm{NMR}\left(\mathrm{CDCl}_{3}, \mathrm{ppm}\right): \delta 74.5\left(\mathrm{PPh}_{3}\right) . \mathrm{UV} / \mathrm{vis}$, $\lambda_{\max }(\epsilon)$ : 342 (5532), 508 (1691). Anal. Calcd for $\mathrm{C}_{31} \mathrm{H}_{34} \mathrm{~F}_{6} \mathrm{~N}_{3} \mathrm{O}_{6}$ $\mathrm{PRuS}_{2}$ : C, 43.56; H, 4.01; N, 4.92. Found: C, 43.42; H, 4.08; N, 4.86.

Synthesis of $\left[\right.$ mer-RuCl$\left.(\mathbf{M e C N})\left(\mathbf{N N}^{\prime} \mathbf{N}\right)\left(\mathbf{P P h}_{3}\right)\right] \mathrm{OTf}$ (6a). To a solution of $1(0.16 \mathrm{~g}, 0.25 \mathrm{mmol})$ in $\mathrm{CH}_{2} \mathrm{Cl}_{2}(30 \mathrm{~mL}) / \mathrm{MeCN}(5 \mathrm{~mL})$ was added AgOTf $(0.07 \mathrm{~g}, 0.27 \mathrm{mmol})$. After the reaction mixture was stirred for $30 \mathrm{~min}$, a newly formed flaky white precipitate was filtered off. After the solvent was removed in vacuo, the crude product was extracted with $\mathrm{Et}_{2} \mathrm{O}(10 \mathrm{~mL}, 2 \times)$, leaving the pure product as a light brown solid $(0.18 \mathrm{~g}, 90 \%)$. No decomposition at $T<200{ }^{\circ} \mathrm{C} .{ }^{1} \mathrm{H}$ NMR $\left(\mathrm{CDCl}_{3}, \mathrm{ppm}\right): \delta 8.13\left(\mathrm{t}, 6 \mathrm{H}, o-\mathrm{H} \mathrm{PPh}_{3}\right), 7.87(\mathrm{t}, 1 \mathrm{H}, p-\mathrm{H}$ py), $7.47\left(\mathrm{~d}, 2 \mathrm{H}, m-\mathrm{H}\right.$ py), 7.44-7.36 (m, 9H, ar-H), $4.30\left(\mathrm{~d}, 2 \mathrm{H},{ }^{2} J_{\mathrm{HAHB}}=\right.$ $\left.15 \mathrm{~Hz}, \mathrm{CH}_{A} \mathrm{H}_{\mathrm{B}}\right), 3.89\left(\mathrm{~d}, 2 \mathrm{H},{ }^{2} J_{\mathrm{HAHB}}=15 \mathrm{~Hz}, \mathrm{CH}_{\mathrm{A}} H_{B}\right), 2.37(\mathrm{~s}, 6 \mathrm{H}$, $\mathrm{N}(M e)(\mathrm{Me})), 2.11(\mathrm{~s}, 6 \mathrm{H}, \mathrm{N}(\mathrm{Me})(\mathrm{Me})), 1.91(\mathrm{~s}, 3 \mathrm{H}, \mathrm{MeCN}) .{ }^{13} \mathrm{C} \mathrm{NMR}$ $\left(\mathrm{CDCl}_{3}, \mathrm{ppm}\right): \delta 159.6\left(o-\mathrm{C}\right.$ py), $137.8\left(p-\mathrm{C}\right.$ py), $136.1\left(\mathrm{~d},{ }^{1} J_{\mathrm{PC}}=\right.$ $35.5 \mathrm{~Hz}$, ipso-C $\left.\mathrm{PPh}_{3}\right), 133.0\left(\mathrm{~d},{ }^{3} J_{\mathrm{PC}}=9.8 \mathrm{~Hz}, m-\mathrm{C} \mathrm{PPh}_{3}\right), 129.4(p-\mathrm{C}$ $\left.\mathrm{PPh}_{3}\right), 128.6\left(\mathrm{~d},{ }^{2} J_{\mathrm{PC}}=8.8 \mathrm{~Hz}, o-\mathrm{C} \mathrm{PPh}\right)_{3}, 127.3(\mathrm{MeCN}), 121.0(m-\mathrm{C}$ py), $73.6\left(\mathrm{CH}_{2}\right), 55.7(\mathrm{~N}(\mathrm{Me})(\mathrm{Me})), 55.1(\mathrm{~N}(\mathrm{Me})(\mathrm{Me})), 4.1(\mathrm{MeCN})$. ${ }^{31} \mathrm{P} \mathrm{NMR}\left(\mathrm{CDCl}_{3}, \mathrm{ppm}\right): \delta 32.8\left(\mathrm{PPh}_{3}\right)$. UV/vis, $\lambda_{\max }(\epsilon): 350(4663)$. Anal. Calcd for $\mathrm{C}_{32} \mathrm{H}_{37} \mathrm{ClF}_{3} \mathrm{~N}_{4} \mathrm{O}_{3} \mathrm{PRuS}$ : C, 49.13; H, 4.77; N, 7.16. Found: $\mathrm{C}, 48.96 ; \mathrm{H}, 4.72 ; \mathrm{N}, 7.06$. 
Synthesis of $\left[\right.$ mer $\left.-\mathrm{RuCl}(\mathrm{MeCN})\left(\mathrm{NN}^{\prime} \mathbf{N}\right)\left(\mathbf{P P h}_{3}\right)\right] \mathrm{Cl}(\mathbf{6 b})$. A suspension of $\mathbf{1}(0.31 \mathrm{~g}, 0.49 \mathrm{mmol})$ in acetonitrile $(30 \mathrm{~mL})$ was gently heated to $55{ }^{\circ} \mathrm{C}$ for $5 \mathrm{~min}$, upon which a clear brown solution was formed. After $\mathrm{MeCN}$ was evaporated, the crude product was dissolved in $\mathrm{CHCl}_{3}$ $(25 \mathrm{~mL})$. After pentane $(50 \mathrm{~mL})$ was added, the pure product was collected by centrifugation as a cream-colored solid $(0.30 \mathrm{~g}, 91 \%) .{ }^{1} \mathrm{H}$ NMR $\left(\mathrm{CDCl}_{3}, \mathrm{ppm}\right): \delta 8.13(\mathrm{t}, 6 \mathrm{H}, o-\mathrm{H}$ py), $7.84(\mathrm{t}, 1 \mathrm{H}, p-\mathrm{H}$ py), $7.53\left(\mathrm{~d}, 2 \mathrm{H}, m-\mathrm{H}\right.$ py), 7.44-7.35 (m, 9H, ar-H py), $4.33\left(\mathrm{~d}, 2 \mathrm{H},{ }^{2} J_{\mathrm{HAHB}}\right.$ $\left.=15.5 \mathrm{~Hz}, \mathrm{CH}_{A} \mathrm{H}_{\mathrm{B}}\right), 4.12\left(\mathrm{~d}, 2 \mathrm{H},{ }^{2} J_{\mathrm{HAHB}}=15.5 \mathrm{~Hz}, \mathrm{CH}_{\mathrm{A}} H_{B}\right), 2.36(\mathrm{~s}$, $6 \mathrm{H}, \mathrm{N}(\mathrm{Me})(\mathrm{Me})), 2.15(\mathrm{~s}, 3 \mathrm{H}, \mathrm{MeCN}), 2.12(\mathrm{~s}, 6 \mathrm{H}, \mathrm{N}(\mathrm{Me})(M e)) .{ }^{13} \mathrm{C}$ $\mathrm{NMR}\left(\mathrm{CDCl}_{3}, \mathrm{ppm}\right): \delta 159.7$ (o-C py), $137.6\left(p-\mathrm{C}\right.$ py), $136.1\left(\mathrm{~d},{ }^{1} J_{\mathrm{PC}}\right.$ $=35.1 \mathrm{~Hz}$, ipso-C $\left.\mathrm{PPh}_{3}\right), 133.0\left(\mathrm{~d},{ }^{3} J_{\mathrm{PC}}=9.3 \mathrm{~Hz}, m-\mathrm{C} \mathrm{PPh}\right), 129.3$ $\left(p-\mathrm{C} \mathrm{PPh}{ }_{3}\right), 128.6\left(\mathrm{~d},{ }^{2} J_{\mathrm{PC}}=8.8 \mathrm{~Hz}, o-\mathrm{C} \mathrm{PPh}{ }_{3}\right), 127.6(\mathrm{Me} C N), 121.0$ $\left(m-\mathrm{C}\right.$ py), $73.9\left(\mathrm{CH}_{2}\right), 55.8(\mathrm{~N}(\mathrm{Me})(\mathrm{Me})), 55.3(\mathrm{~N}(\mathrm{Me})(M e)), 5.6$ $(\mathrm{MeCN}) .{ }^{31} \mathrm{P} \mathrm{NMR}\left(\mathrm{CDCl}_{3}, \mathrm{ppm}\right): \delta 33.0\left(\mathrm{PPh}_{3}\right)$. UV/vis, $\lambda_{\max }(\epsilon)$ : 350 (4609). Anal. Calcd for $\mathrm{C}_{31} \mathrm{H}_{37} \mathrm{Cl}_{2} \mathrm{~N}_{4} \mathrm{PRu} \cdot 3 / 2 \mathrm{CH}_{2} \mathrm{Cl}_{2}$ : C, 49.04; H, 5.07; N, 7.04. Found: C, 49.36; H, 5.10; N, 7.27.

Synthesis of $\left[\right.$ mer,trans $\left.-\mathrm{Ru}(\mathrm{MeCN})_{2}\left(\mathrm{NN}^{\prime} \mathbf{N}\right)\left(\mathbf{P P h}_{3}\right)\right] \mathrm{OTf}_{2}(\mathbf{7})$. To a solution of $1(0.75 \mathrm{~g}, 1.2 \mathrm{mmol})$ in $\mathrm{CH}_{2} \mathrm{Cl}_{2}(30 \mathrm{~mL}) / \mathrm{MeCN}(5 \mathrm{~mL})$ was added $\operatorname{AgOTf}(0.61 \mathrm{~g}, 2.4 \mathrm{mmol})$. After the suspension was stirred for $30 \mathrm{~min}$, the newly formed flaky white precipitate was filtered off. After the solvent was removed in vacuo and the residue had been extracted with $\mathrm{Et}_{2} \mathrm{O}(10 \mathrm{~mL}, 2 \times)$, the pure product was obtained as a yellow solid $(0.97 \mathrm{~g}, 86 \%)$. No decomposition at $T<200{ }^{\circ} \mathrm{C}$. ${ }^{1} \mathrm{H}$ NMR (DMSO- $\left.d_{6}, \mathrm{ppm}\right): \delta 8.11(\mathrm{t}, 1 \mathrm{H}, p-\mathrm{H}$ py), 7.77-7.71 $(\mathrm{m}, 8 \mathrm{H}$, ar-H), 7.55-7.46 (m, 9H, ar-H), 4.14 (s, 4H, $\left.\mathrm{CH}_{2}\right), 2.41$ (s, 6H, MeCN), $2.20\left(\mathrm{~s}, 12 \mathrm{H}, \mathrm{NMe}_{2}\right) .{ }^{13} \mathrm{C} \mathrm{NMR}$ (DMSO- $\left.d_{6}, \mathrm{ppm}\right): \delta 161.4$ (o-C py), 141.8 (p-C py), $136.5\left(\mathrm{~d},{ }^{1} J_{\mathrm{PC}}=37.9 \mathrm{~Hz}\right.$, ipso-C $\left.\mathrm{PPh}_{3}\right), 135.0\left(\mathrm{~d},{ }^{3} J_{\mathrm{PC}}\right.$ $=9.8 \mathrm{~Hz}, m-\mathrm{C} \mathrm{PPh}), 132.5\left(p-\mathrm{C} \mathrm{PPh}_{3}\right), 132.3(\mathrm{MeCN}), 123.2\left(\mathrm{q},{ }^{1} J_{\mathrm{CF}}\right.$ $\left.=322 \mathrm{~Hz}, \mathrm{SO}_{3} \mathrm{CF}_{3}\right), 75.1\left(\mathrm{CH}_{2}\right), 57.2\left(\mathrm{NMe}_{2}\right), 6.73(\mathrm{MeCN}) .{ }^{31} \mathrm{P} \mathrm{NMR}$ (DMSO- $\left.d_{6}, \mathrm{ppm}\right): \delta 37.4\left(\mathrm{PPh}_{3}\right)$. UV/vis, $\lambda_{\max }(\epsilon): 282$ (5933). Anal. Calcd for $\mathrm{C}_{35} \mathrm{H}_{40} \mathrm{~F}_{6} \mathrm{~N}_{5} \mathrm{O}_{6} \mathrm{PRuS}_{2}$ : C, 44.87; $\mathrm{H}, 4.30 ; \mathrm{N}, 7.47$. Found: C, 44.72; H, 4.31; N, 7.38.

Synthesis of $\left[\left(\boldsymbol{\mu}-\mathbf{N}_{2}\right)\left(\text { mer,trans }-\mathbf{R u C l}_{2}\left(\mathbf{N N}^{\prime} \mathbf{N}\right)\right)_{2}\right](8)$. To a suspension of $\left[\mathrm{RuCl}_{2}(\mathrm{nbd})\right]_{n}(1.28 \mathrm{~g}, 4.85 \mathrm{mmol})$ in $\mathrm{C}_{6} \mathrm{H}_{6}(100 \mathrm{~mL})$ was added $\mathbf{N N}^{\prime} \mathbf{N}(1.02 \mathrm{~g}, 5.28 \mathrm{mmol}$ ) at room temperature, after which the reaction mixture was refluxed for $24 \mathrm{~h}$. Subsequently the solvent was removed in vacuo. The resulting residue was extracted with $\mathrm{CH}_{2} \mathrm{Cl}_{2}(20 \mathrm{~mL})$, separated from insoluble starting material, and extracted with pentane $(20 \mathrm{~mL}, 2 \times)$. After the solvent was removed in vacuo, almost pure crude product was obtained in about $60 \%$ yield. Slow diffusion of $\mathrm{Et}_{2} \mathrm{O}$ into a $\mathrm{CH}_{2} \mathrm{Cl}_{2}$ solution of this crude product resulted in the formation of yellow-brown, block-shaped crystals $(1.0 \mathrm{~g}, 30 \%) .{ }^{1} \mathrm{H}$ NMR $\left(\mathrm{CDCl}_{3}, \mathrm{ppm}\right): \delta 7.60(\mathrm{t}, 1 \mathrm{H}, p-\mathrm{H}$ py), $7.27(\mathrm{~d}, 2 \mathrm{H}, m-\mathrm{H}$ py), $4.15\left(\mathrm{~s}, 4 \mathrm{H}, \mathrm{CH}_{2}\right), 2.80\left(\mathrm{~s}, 12 \mathrm{H}, \mathrm{NMe}_{2}\right) .{ }^{13} \mathrm{C} \mathrm{NMR}\left(\mathrm{CDCl}_{3}, \mathrm{ppm}\right): \delta$ 162.5 (o-C py), 134.6 ( $p$-C py), 119.5 ( $m$-C py), $71.7\left(\mathrm{CH}_{2}\right), 55.0$ $\left(\mathrm{NMe}_{2}\right)$. FAB-MS $760.0\left(\mathrm{M}^{+}\right)$. Raman $\left(\mathrm{cm}^{-1}\right): 2099(\mathrm{~N} \equiv \mathrm{N}) . \mathrm{UV} /$ vis, $\lambda_{\max }(\epsilon)$ : 426 (14 632). Anal. Calcd for $\mathrm{C}_{22} \mathrm{H}_{38} \mathrm{Cl}_{4} \mathrm{~N}_{8} \mathrm{Ru}_{2}$ : C, 34.84; H, 5.05; N, 14.77. Found: C, 33.54; H, 4.80; N, 13.27 (incorrect, but $\mathrm{C} / \mathrm{H}, \mathrm{C} / \mathrm{N}$, and $\mathrm{H} / \mathrm{N}$ ratios consistent with $\left.8 \cdot x \mathrm{RuCl}_{y}\right)$.

Synthesis of $\left[\right.$ mer,trans- $\left.\mathbf{R u C l}_{2}(\mathrm{CO})\left(\mathrm{NN}^{\prime} \mathbf{N}\right)\right](9)$. Through a solution of $8(100 \mathrm{mg}, 0.132 \mathrm{mmol})$ in $\mathrm{CH}_{2} \mathrm{Cl}_{2}(20 \mathrm{~mL})$ was bubbled $\mathrm{CO}$ for $90 \mathrm{~min}$ at room temperature, during which the color of the solution changed from green-yellow to light brown. After this, the solvent was removed in vacuo and the residue was extracted with pentane $(10 \mathrm{~mL})$, upon which the pure product remained as a green-brown solid (100 $\mathrm{mg}, 96 \%$, based on 8). ${ }^{1} \mathrm{H} \mathrm{NMR}\left(\mathrm{CD}_{2} \mathrm{Cl}_{2}, \mathrm{ppm}\right): \delta 7.93(\mathrm{t}, 1 \mathrm{H}, p-\mathrm{H}$ py), $7.48\left(\mathrm{~d}, 2 \mathrm{H}, m-\mathrm{H}\right.$ py), $4.22\left(\mathrm{~s}, 4 \mathrm{H}, \mathrm{CH}_{2}\right), 2.68\left(\mathrm{~s}, 12 \mathrm{H}, \mathrm{NMe}_{2}\right) .{ }^{13} \mathrm{C}$ NMR $\left(\mathrm{CD}_{2} \mathrm{Cl}_{2}, \mathrm{ppm}\right): \delta 185.4(\mathrm{CO}), 160.1$ (o-C py), 139.0 ( $m$-C py), 121.0 ( $m$-C py), $72.5\left(\mathrm{CH}_{2}\right), 56.4\left(\mathrm{NMe}_{2}\right)$. IR ( $\left.\mathrm{KBr}\right): v_{\mathrm{CO}} 1948 \mathrm{~cm}^{-1}$. Anal. Calcd for $\mathrm{C}_{12} \mathrm{H}_{19} \mathrm{Cl}_{2} \mathrm{~N}_{3} \mathrm{ORu}$ : C, 36.65; H, 4.87; N, 10.68 . Found: C, 36.52; $\mathrm{H}, 4.85 ; \mathrm{N}, 10.54$.

Synthesis of $\left[m e r, c i s-\operatorname{RuCl}(\mathbf{C O})_{2}\left(\mathbf{N N}^{\prime} \mathbf{N}\right)\right] \mathrm{OTf}(10)$. Through a solution of $3(1.64 \mathrm{~g}, 1.92 \mathrm{mmol})$ in $\mathrm{CH}_{2} \mathrm{Cl}_{2}(10 \mathrm{~mL})$ was bubbled $\mathrm{CO}$ for $4 \mathrm{~min}$, upon which a distinct color change from deep purple to clear yellow was observed. After the reaction mixture was stirred for 30 min under a $\mathrm{CO}$ atmosphere, pentane $(40 \mathrm{~mL})$ was added, upon which a yellow solid precipitated. ${ }^{1} \mathrm{H}$ NMR analysis confirmed that this crude product consisted of about $80 \%$ of $\mathbf{1 0}$ along with $20 \%$ of 11. Further treatment of the crude product with $\mathrm{CO}$ in a $\mathrm{CH}_{2} \mathrm{Cl}_{2}$ solution $(10 \mathrm{~mL})$ did not lead to significant improvement of product distribution. Fractional crystallization by slow diffusion of pentane in a $\mathrm{CH}_{2} \mathrm{Cl}_{2}$ solution of the crude product afforded almost pure product as a light-yellow microcrystalline solid $(0.2 \mathrm{~g}, 20 \%)$. No decomposition at $T<200{ }^{\circ} \mathrm{C}$. ${ }^{1} \mathrm{H}$ NMR $\left(\mathrm{CD}_{3} \mathrm{CN}, \mathrm{ppm}\right): \delta 7.96(\mathrm{t}, 1 \mathrm{H}, p-\mathrm{H}$ py), 7.54 $\left(\mathrm{d}, 2 \mathrm{H}, m-\mathrm{H}\right.$ py), $4.36\left(\mathrm{~d},{ }^{2} J_{\mathrm{HAHB}}=16.2 \mathrm{~Hz}, \mathrm{CH}_{A} \mathrm{H}_{\mathrm{B}}\right), 4.13\left(\mathrm{~d},{ }^{2} J_{\mathrm{HAHB}}\right.$ $\left.=16.2 \mathrm{~Hz}, \mathrm{CH}_{\mathrm{A}} H_{B}\right), 2.84(\mathrm{~s}, 6 \mathrm{H}, \mathrm{N}(\mathrm{Me})(\mathrm{Me})), 2.66(\mathrm{~s}, 6 \mathrm{H}, \mathrm{N}(\mathrm{Me})-$ $(\mathrm{Me})) .{ }^{13} \mathrm{C} \mathrm{NMR}\left(\mathrm{CD}_{3} \mathrm{CN}, \mathrm{ppm}\right): \delta 206.42,200.98(\mathrm{CO}), 160.92(o-\mathrm{C}$ py), 139.61 ( $p$-C py), 122.52 (m-C py), $74.24\left(\mathrm{CH}_{2}\right), 58.36(\mathrm{~N}(\mathrm{Me})-$ (Me)), $54.27(\mathrm{~N}(\mathrm{Me})(\mathrm{Me}))$. IR (KBr): $v_{\mathrm{CO}} 1967,1936 \mathrm{~cm}^{-1}$. Anal. Calcd for $\mathrm{C}_{14} \mathrm{H}_{19} \mathrm{ClF}_{3} \mathrm{~N}_{3} \mathrm{O}_{5} \mathrm{RuS}$ : C, 31.43; $\mathrm{H}, 3.58 ; \mathrm{N}, 7.86$. Found: C, 26.40; H, 3.85; N, 6.63.

Synthesis of $\left[\right.$ mer-RuCl$\left.(\mathbf{C O})\left(\mathbf{N N}^{\prime} \mathbf{N}\right)\left(\mathbf{P P h}_{3}\right)\right] \mathrm{OTf}(11)$. Through a solution of $3(0.8 \mathrm{~g}, 1.15 \mathrm{mmol})$ in $\mathrm{CH}_{2} \mathrm{CH}_{2}(10 \mathrm{~mL})$ was bubbled $\mathrm{CO}$ until the purple color of the solution had disappeared and the reaction mixture had turned light brown (2-3 min). Slow diffusion of pentane into the reaction mixture gave the pure product as large pale yellowbrown crystals which proved to contain 1 equiv of dichloromethane $(0.82 \mathrm{~g}, 83 \%) .{ }^{1} \mathrm{H}$ NMR $\left(\mathrm{CD}_{2} \mathrm{Cl}_{2}, \mathrm{ppm}\right): \delta 8.22-8.10(\mathrm{~m}, 6 \mathrm{H}, o-\mathrm{H}$ $\left.\mathrm{PPh}_{3}\right), 8.03$ (t, $1 \mathrm{H}, p-\mathrm{H}$ py), 7.65 (d, 2H, $m-\mathrm{H}$ py), 7.51-7.48 (m, 9H, ar-H), $4.63\left(\mathrm{~d}, 2 \mathrm{H},{ }^{2} J_{\mathrm{HAHB}}=15.7 \mathrm{~Hz}, \mathrm{CH}_{\mathrm{A}} \mathrm{H}_{\mathrm{B}}\right), 3.97\left(\mathrm{~d}, 2 \mathrm{H},{ }^{2} J_{\mathrm{HAHB}}=\right.$ $\left.15.7 \mathrm{~Hz}, \mathrm{CH}_{\mathrm{A}} H_{B}\right), 2.50(\mathrm{~s}, 6 \mathrm{H}, \mathrm{N}(M e)(\mathrm{Me})), 2.48(\mathrm{~s}, 6 \mathrm{H}, \mathrm{N}(\mathrm{Me})(M e))$. ${ }^{13} \mathrm{C}$ NMR $\left(\mathrm{CD}_{2} \mathrm{Cl}_{2}, \mathrm{ppm}\right): \delta 200.52\left(\mathrm{~d},{ }^{2} J_{\mathrm{PC}}=13.6 \mathrm{~Hz}, \mathrm{CO}\right), 159.78$ (o-C py), 142.13 (p-C py), $136.01\left(\mathrm{~d},{ }^{1} J_{\mathrm{PC}}=41.4 \mathrm{~Hz}\right.$, ipso-C $\mathrm{PPh}_{3}$ ), $134.73\left(\mathrm{~d},{ }^{3} J_{\mathrm{PC}}=8.9 \mathrm{~Hz}, m-\mathrm{C} \mathrm{PPh}\right), 132.58\left(\mathrm{~d},{ }^{4} J_{\mathrm{PC}}=1.9 \mathrm{~Hz}, p-\mathrm{C}\right.$ $\left.\mathrm{PPh}_{3}\right), 131.04\left(\mathrm{~d},{ }^{2} J_{\mathrm{PC}}=9.4 \mathrm{~Hz}, o-\mathrm{C} \mathrm{PPh}_{3}\right), 123.92(m-\mathrm{C}$ py), 76.66 $\left(\mathrm{CH}_{2}\right), 60.46(\mathrm{~N}(\mathrm{Me})(\mathrm{Me})), 57.11(\mathrm{~N}(\mathrm{Me})(\mathrm{Me})) .{ }^{31} \mathrm{P} \mathrm{NMR}\left(\mathrm{CD}_{2} \mathrm{Cl}_{2}\right.$, $\mathrm{ppm}): \delta 17.31\left(\mathrm{PPh}_{3}\right)$. IR $(\mathrm{KBr}): v_{\mathrm{CO}} 1964 \mathrm{~cm}^{-1}$. UV/vis, $\lambda_{\max }(\epsilon)$ : 238 (39 950). Anal. Calcd for $\mathrm{C}_{31} \mathrm{H}_{34} \mathrm{ClF}_{3} \mathrm{~N}_{3} \mathrm{O}_{4} \mathrm{PRuS} \cdot \mathrm{CH}_{2} \mathrm{Cl}_{2}$ : C, 45.00; H, 4.25; N, 4.92. Found: C, 44.64; H, 4.18; N, 4.87.

$[$ mer,cis-RuOTf(CO)(NN'N)(PPh $)]$ OTf (12). ${ }^{1} \mathrm{H}$ NMR $\left(\mathrm{CDCl}_{3}\right.$, $\mathrm{ppm}): \delta 7.95(\mathrm{t}, 1 \mathrm{H}, p-\mathrm{H}$ py), $7.50(\mathrm{~d}, 2 \mathrm{H}, m-\mathrm{H}$ py), 7.45-7.05 (m, $15 \mathrm{H}$, ar-H), $4.58\left(\mathrm{~d}, 2 \mathrm{H},{ }^{2} J_{\mathrm{HAHB}}=16.5 \mathrm{~Hz}, \mathrm{CH}_{A} \mathrm{H}_{\mathrm{B}}\right), 4.11\left(\mathrm{~d}, 2 \mathrm{H},{ }^{2} J_{\mathrm{HAHB}}\right.$ $\left.=16.5 \mathrm{~Hz}, \mathrm{CH}_{\mathrm{A}} H_{B}\right), 2.97(\mathrm{~s}, 6 \mathrm{H}, \mathrm{N}(\mathrm{Me})(\mathrm{Me})), 2.76(\mathrm{~s}, 6 \mathrm{H}, \mathrm{N}(\mathrm{Me})-$ $(\mathrm{Me})) .{ }^{31} \mathrm{P} \mathrm{NMR}\left(\mathrm{CDCl}_{3}, \mathrm{ppm}\right): \delta 7.94\left(\mathrm{PPh}_{3}\right) . \mathrm{IR}(\mathrm{KBr}): v_{\mathrm{CO}} 1964$ $\mathrm{cm}^{-1}$.

Synthesis of $\left[m e r-\operatorname{RuOTf}(\mathrm{CO})\left(\mathrm{NN}^{\prime} \mathbf{N}\right)(\mathrm{HOEt})\right] \mathrm{OTf}(13)$. To a solution of $4(1.0 \mathrm{~g}, 1.17 \mathrm{mmol})$ in a mixture of $\mathrm{CH}_{2} \mathrm{Cl}_{2}(8 \mathrm{~mL})$ and $\mathrm{EtOH}(2 \mathrm{~mL})$ was bubbled $\mathrm{CO}$ for $2 \mathrm{~min}$, during which the color of the solution changed from purple to clear light yellow. Slow diffusion of pentane into the reaction mixture afforded the pure product as large yellow to orange block-shaped crystals $(0.3 \mathrm{~g}, 38 \%)$. No NMR data could be obtained due to instability and/or insolubility in different deuterated solvents. IR (KBr): $v_{\mathrm{CO}} 1968 \mathrm{~cm}^{-1}$. UV/vis, $\lambda_{\max }(\epsilon): 230$ (7700), 264 (5287). Anal. Calcd for $\mathrm{C}_{16} \mathrm{H}_{25} \mathrm{~F}_{6} \mathrm{~N}_{3} \mathrm{O}_{8} \mathrm{RuS}_{2}$ : C, 28.83; H, 3.78; N, 6.30. Found: C, 28.76; H, 3.86; N, 6.25.

Substitutional Reactivity of $\left[\right.$ mer,trans $\left.-\mathbf{R u C l}_{2}\left(\mathbf{N N}^{\prime} \mathbf{N}\right)\left(\mathbf{P P h}_{3}\right)\right](1)$ toward $\mathrm{PMe}_{3}$. To a solution of $\mathbf{1}(0.45 \mathrm{~g}, 0.72 \mathrm{mmol})$ in $\mathrm{CH}_{2} \mathrm{Cl}_{2}(50$ $\mathrm{mL})$ was added a solution of $\mathrm{PMe}_{3}(133 \mathrm{mg}, 1.75 \mathrm{mmol})$ in $\mathrm{MeOH}$ $(2.5 \mathrm{~mL})$, which led to a slight color change from brown to reddish brown. ${ }^{31} \mathrm{P}\left\{{ }^{1} \mathrm{H}\right\}$ and ${ }^{1} \mathrm{H}$ NMR of the resulting solution indicated that $\mathrm{RuCl}_{2}\left(\mathrm{PMe}_{3}\right)_{4}$ (by comparison with an authentic sample ${ }^{16}$ ) had been formed. ${ }^{1} \mathrm{H}$ NMR $\left(\mathrm{CDCl}_{3}, \mathrm{ppm}\right): \delta 1.50\left(\mathrm{~s}, \mathrm{PMe}_{3}\right),{ }^{13} \mathrm{C} \mathrm{NMR}\left(\mathrm{CDCl}_{3}\right.$, $\mathrm{ppm}): \delta 17.42\left(\mathrm{~m}, \mathrm{PMe}_{3}\right) .{ }^{31} \mathrm{P} \mathrm{NMR}\left(\mathrm{CDCl}_{3}, \mathrm{ppm}\right): \delta-5.95$.

\section{Crystal Structure Determinations}

All crystals were sampled and mounted with the oil technique and cut to size when needed. X-ray data were collected at $150 \mathrm{~K}$ on an Enraf-Nonius CAD4T on a rotating anode using graphite-monochromated Mo $\mathrm{K} \alpha$ radiation. Unit cell parameters were derived from the SET $4{ }^{19}$ setting angles of 25 reflections. Unit cells were checked for higher symmetry with the programs LEPAGE ${ }^{20}$ and PLATON/ MISSYM. ${ }^{21}$ All geometrical calculations and the ORTEP illustrations were done with PLATON ${ }^{22}$ (Table 1).

[mer,trans- $\left.\mathbf{R u C l}_{\mathbf{2}}\left(\mathbf{N N}^{\prime} \mathbf{N}\right)\left(\mathbf{P P h}_{3}\right)\right]$ (1). A total of 23464 reflections $\left(\theta_{\max }=25^{\circ}\right)$ were scanned and averaged into a unique set of 5795

(19) (a) de Boer, J. L.; Duisenberg, A. J. M. Acta Crystallogr. 1984, A40, C410. (b) Le Page, Y. J. Appl. Crystallogr. 1987, 20, 264-269.

(20) Spek, A. L. J. Appl. Crystallogr. 1988, 21, 578-579.

(21) Spek, A. L. Am. Cryst. Assoc. 1997 (Abstract)

(22) Spek, A. L. Acta Crystallogr. 1990, A46, C34. 
Table 1. Experimental Data for the X-ray Diffraction Studies of 1, 4, 6a, 8, 11, and 13

\begin{tabular}{|c|c|c|c|c|c|c|}
\hline & 1 & 4 & $6 \mathbf{a}$ & 8 & 11 & 13 \\
\hline empirical formula & $\begin{array}{c}\mathrm{C}_{29} \mathrm{H}_{34} \mathrm{Cl}_{2} \mathrm{~N}_{3} \mathrm{PRu} \cdot \\
3 / 2 \mathrm{CH}_{2} \mathrm{Cl}_{2}\end{array}$ & $\begin{array}{c}\mathrm{C}_{30} \mathrm{H}_{34} \mathrm{~F}_{3} \mathrm{~N}_{3} \mathrm{O}_{3} \mathrm{PRuS} \cdot \\
\mathrm{CF}_{3} \mathrm{SO}_{3} \cdot \mathrm{CH}_{2} \mathrm{Cl}_{2}\end{array}$ & $\begin{array}{l}\mathrm{C}_{31} \mathrm{H}_{37} \mathrm{ClN}_{4} \mathrm{PRu} \cdot \\
\quad \mathrm{CF}_{3} \mathrm{SO}_{3} \cdot 2.75 \mathrm{CHCl}_{3}\end{array}$ & $\begin{array}{c}\mathrm{C}_{22} \mathrm{H}_{38} \mathrm{Cl}_{4} \mathrm{~N}_{8} \mathrm{Ru}_{2} \cdot \\
{ }_{1 / 2} \mathrm{C}_{4} \mathrm{H}_{10} \mathrm{O}\end{array}$ & $\begin{array}{l}\mathrm{C}_{30} \mathrm{H}_{34} \mathrm{ClN}_{3} \mathrm{OPRu} \cdot \\
\mathrm{CF}_{3} \mathrm{SO}_{3} \cdot{ }^{\circ} / \mathrm{CH}_{2} \mathrm{Cl}_{2}\end{array}$ & $\begin{array}{l}\mathrm{C}_{15} \mathrm{H}_{25} \mathrm{~F}_{3} \mathrm{~N}_{3} \mathrm{O}_{5} \mathrm{RuS} \cdot \\
\mathrm{CF}_{3} \mathrm{SO}_{3}\end{array}$ \\
\hline fw & 754.96 & 939.73 & 1110.51 & 795.61 & 875.35 & 666.58 \\
\hline color & orange & red & yellow & red & yellowish & yellowish \\
\hline space group & $F d d 2$ (No. 43) & $P \overline{1}($ No. 2$)$ & $P \overline{1}$ (No. 2) & $P 2_{1} / c$ (No. 14) & $P 2_{1} 2_{1} 2_{1}$ (No. 19) & $P 2_{1}$ (No. 4) \\
\hline$a(\AA)$ & $23.472(9)$ & $9.3763(10)$ & $12.4610(8)$ & $14.8201(12)$ & $12.335(1)$ & $10.169(1)$ \\
\hline$b(\AA)$ & $59.277(13)$ & $13.484(5)$ & $17.460(2)$ & $14.0198(12)$ & $21.523(2)$ & $10.313(1)$ \\
\hline$c(\AA)$ & $9.4320(10)$ & $15.946(3)$ & $22.737(2)$ & $15.1417(11)$ & $27.393(2)$ & $12.214(1)$ \\
\hline$\alpha(\mathrm{deg})$ & - & $84.23(2)$ & $111.485(9)$ & - & - & - \\
\hline$\beta(\operatorname{deg}$ & - & $75.469(11)$ & $91.413(6)$ & $93.243(5)$ & - & $101.19(1)$ \\
\hline$\gamma(\operatorname{deg})$ & - & $78.13(2)$ & $97.951(7)$ & - & - & - \\
\hline$V\left(\AA^{3}\right)$ & 13123(6) & 1907.3(9) & $4543.6(8)$ & $3141.0(4)$ & $7272(1)$ & $1256.6(2)$ \\
\hline$\lambda(\operatorname{Mo~K} \alpha)(\AA)$ & 0.71073 & 0.71073 & 0.71073 & 0.71073 & 0.71073 & 0.71073 \\
\hline$Z$ & 16 & 2 & 4 & 4 & 8 & 2 \\
\hline$d_{\text {calc }}\left(\mathrm{g} \cdot \mathrm{cm}^{-3}\right)$ & 1.528 & 1.636 & 1.623 & 1.682 & 1.599 & 1.762 \\
\hline$\mu(\mathrm{Mo} \mathrm{K} \alpha)\left(\mathrm{cm}^{-1}\right)$ & 9.6 & 7.7 & 10.11 & 13.09 & 8.36 & 8.8 \\
\hline $\mathrm{R}_{1}[I>2 \sigma(I)]^{a}$ & 0.0738 & 0.0720 & 0.0529 & 0.0297 & 0.0589 & 0.0231 \\
\hline $\mathrm{wR}_{2}$ & 0.1799 & 0.1893 & 0.1436 & 0.0771 & 0.1263 & 0.0593 \\
\hline
\end{tabular}

$$
{ }^{a} \mathrm{R}_{1}=\sum\left(F_{\mathrm{o}}-\left|F_{\mathrm{c}}\right|\right) / \sum F_{\mathrm{o}} ; \mathrm{wR}_{2}=\left[\sum w\left(F_{\mathrm{o}}{ }^{2}-F_{\mathrm{c}}{ }^{2}\right)^{2} / \sum w\left(F_{\mathrm{o}}{ }^{2}\right)^{2}\right]^{1 / 2} .
$$

reflections (5083 with $I>\sigma(I)$ ). The structure was solved by automated Patterson methods (DIRDIF ${ }^{23}$ ) and refined on $F^{2}$ with SHELXL93. ${ }^{24}$ Hydrogen atoms were taken into account at calculated positions.

[RuOTf(NN'N)(PPh $\left.\mathbf{R}_{3}\right)$ ]OTf (4). A total of 7294 reflections $\left(\theta_{\max }\right.$ $\left.=25.4^{\circ}\right)$ were scanned and averaged into a unique set of 6851 reflections (4903 with $I>2 \sigma(I)$ ). The structure was solved by automated Patterson methods (DIRDIF ${ }^{23}$ ) and refined on $F^{2}$ with SHELXL93. ${ }^{24}$ No satisfactory disorder model for the $\mathrm{CH}_{2} \mathrm{Cl}_{2}$ solvent molecules of crystallization at $0.5,0.5,0.5$ could be refined. Therefore, its contribution in the structure factor calculations was taken into account using the PLATON/SQUEEZE ${ }^{25,26}$ method. Hydrogen atoms were taken into account at calculated positions.

[mer-RuCl(MeCN)(NN'N)(PPh$\left.\left.{ }_{3}\right)\right]$ OTf (6a). A total of 16898 reflections $\left(\theta_{\max }=25.4^{\circ}\right)$ were scanned and averaged into a unique set of 16116 reflections (11985 with $I>2 \sigma(I)$ ). The structure was solved by direct methods (SHELXS86 ${ }^{27}$ ) and refined on $F^{2}$ with SHELXL93. ${ }^{24}$ Disordered solvents $\left(\mathrm{CHCl}_{3}\right)$ were taken into account using PLATON/SQUEEZE. ${ }^{25,26}$ Hydrogen atoms were taken into account at calculated positions.

$\left[\left(\boldsymbol{\mu}-\mathbf{N}_{2}\right)\left(\text { mer,trans }-\mathbf{R u C l}_{2}\left(\mathbf{N N}^{\prime} \mathbf{N}\right)\right)_{2}\right](\mathbf{8})$. A total of 19600 reflections $\left(\theta_{\max }=27.5^{\circ}\right)$ were scanned and averaged into a unique set of 7205 reflections $(6402$ with $I>2 \sigma(I))$. The structure was solved by direct methods (SHELXS86 ${ }^{27}$ ) and refined on $F^{2}$ with SHELXL96. ${ }^{28}$ Disordered diethyl ether was handled with the PLATON/SQUEEZE ${ }^{25,26}$ technique. Hydrogen atoms were taken into account at calculated positions.

$\left[\right.$ mer-RuCl$\left.(\mathbf{C O})\left(\mathbf{N N}^{\prime} \mathbf{N}\right)\left(\mathbf{P P h}_{3}\right)\right]$ OTf (11). A total of 9670 reflections $\left(\theta_{\max }=27.5^{\circ}\right)$ were scanned and averaged into a unique set of 9571 reflections (6554 with $I>2 \sigma(I))$. The structure was solved by Patterson methods $\left(\right.$ DIRDIF $\left.^{23}\right)$ and refined on $F^{2}$ with SHELXL96. ${ }^{28}$ One of the $\mathrm{SO}_{3} \mathrm{CF}_{3}$ ions is heavily disordered and was taken into account along with the disordered solvent with the PLATON/SQUEEZE ${ }^{25,26}$ technique. Hydrogen atoms were taken into account at calculated positions. The racemic twin parameter refined to $0.41(6)$.

[mer-RuOTf(CO)(NN'N)(HOEt)]OTf (13). A total of 4100 reflections $\left(\theta_{\max }=27.5^{\circ}\right)$ were scanned and averaged into a unique set of 3036 reflections ( 2982 with $I>2 \sigma(I)$ ). The structure was solved by

(23) Beurskens, P. T.; Admiraal, G.; Beurskens, G.; Bosman, W. P.; GarciaGranda, S.; Gould, R. O.; Smits, J. M. M.; Smykala, C. The DIRDIF96 Program System; Technical Report of the Crystallographic Laboratory, Universiteit of Nijmegen: Nijmegen, The Netherlands, 1996.

(24) Sheldrick, G. M. SHELXL93. Program for Crystal Structure Refinement; University of Göttingen: Göttingen, Germany, 1993.

(25) Spek, A. L. Am. Cryst. Assoc. Abstr. 1994, 22, 66.

(26) Spek, A. L. Acta Crystallogr. 1987, C43, 1233-1235.

(27) Sheldrick, G. M. SHELXS86. Program for Crystal Structure Solution; University of Göttingen: Göttingen, Germany, 1986.

(28) Sheldrick, G. M. SHELXS96. Program for Crystal Structure Solution; University of Göttingen: Göttingen, Germany, 1996. direct methods with $\operatorname{SIR}^{29}$ and refined on $F^{2}$ with SHELXL96. ${ }^{28}$ Hydrogen atoms were taken into account at calculated positions. The racemic twin parameter for this model refined to $0.05(3)$.

\section{Results and Discussion}

The synthesis and reaction routes of the new $\mathbf{E N}^{\prime} \mathbf{E}-$ ruthenium(II) $(\mathbf{E}=\mathbf{N}, \mathbf{P})$ complexes are presented in Scheme 1. Relevant spectroscopic data for the complexes are given in Tables 2 and 3.

Synthesis of [mer,trans- $\left.\mathrm{RuCl}_{2}\left(\mathrm{NN}^{\prime} \mathbf{N}\right)\left(\mathrm{PPh}_{3}\right)\right](1)$ and $[m e r-$ ,trans- $\left.\mathbf{R u C l}_{2}(\mathbf{P N P})\left(\mathbf{P P h}_{3}\right)\right]$ (2). The dichloride complex $\left[\mathrm{RuCl}_{2^{-}}\right.$ $\left(\mathbf{N N}^{\prime} \mathbf{N}\right)\left(\mathrm{PPh}_{3}\right)$ ] (1) is conveniently prepared by a ligand exchange reaction of $\left[\mathrm{RuCl}_{2}\left(\mathrm{PPh}_{3}\right)_{3}\right]$ with a stoichiometric amount of $\mathbf{N} \mathbf{N}^{\prime} \mathbf{N}$ in $\mathrm{CH}_{2} \mathrm{Cl}_{2}$. The complex is a yellow-brown solid, stable in both the solid state and in oxygen-free solutions. The retention of one $\mathrm{PPh}_{3}$ ligand in $\mathbf{1}$ is clearly shown by elemental analysis and NMR spectroscopy. In principle, the octahedral complex $\mathbf{1}$ has four stereoisomers. In addition to one trans-dichloro isomer, the cis-dichloro isomer may exist in three diastereomeric forms with mer or fac arrangement of the NN'N donor atoms, made possible by the flexibility of the $o-\mathrm{CH}_{2} \mathrm{NMe}_{2}$ substituents (Chart 1).

The ${ }^{1} \mathrm{H}$ and ${ }^{31} \mathrm{P}$ NMR spectra of $\mathbf{1}$ show a single resonance pattern at all temperatures for all nuclei, indicating the presence of a single stereoisomer in solution. Singlet resonances are observed for the benzylic protons as well as for the $\mathrm{NMe}_{2}$ groups, which point to homotopic benzylic protons and $\mathrm{NMe}_{2}$ groups, respectively. Moreover, the ${ }^{31} \mathrm{P}\left\{{ }^{1} \mathrm{H}\right\}$ NMR shows one high-field singlet resonance for $\mathrm{PPh}_{3}$ at $37.0 \mathrm{ppm}$. This is a characteristic chemical shift for coordinated $\mathrm{PPh}_{3}$ trans to a neutral donor ligand. These observations rule out the cis stereoisomers III and IV (of Chart 1) having a fac arrangement but are in agreement with a mer,trans-I structure, analogous to that established for $\left[\mathrm{RuCl}_{2}\right.$ (terpy) $\left.\left(\mathrm{PPh}_{3}\right)\right]$ (terpy $=2,2,6: 2^{\prime \prime}$ terpyridine) ${ }^{30}$ Of the latter complex, both the mer,trans and the mer,cis isomers can be synthesized. ${ }^{30}$ The quantitative formation of $\left[\right.$ mer, cis- $\mathrm{RuCl}_{2}$ (terpy) $\left.\left(\mathrm{PPh}_{3}\right)\right]$ by a direct ligand exchange reaction of $\mathrm{RuCl}_{2}\left(\mathrm{PPh}_{3}\right)_{3}$ with terpy in benzene at reflux for $1 \mathrm{~h}$ has been reported. ${ }^{30}$ However, the analogous reaction with $\mathbf{N N}^{\prime} \mathbf{N}$ results in an almost quantitative formation

(29) Altomare, A.; Cascarano, G.; Giacovazzo, C.; Guagliardi, A. J. Appl. Crystallogr. 1993, 26, 343-350.

(30) Sullivan, B. P.; Calvert, J. M.; Meyer, T. J. Inorg. Chem. 1980, 19, $1404-1407$. 
Scheme 1. Reaction Routes to $\mathrm{Ru}^{\mathrm{II}}[\mathbf{E N E}]$ Complexes

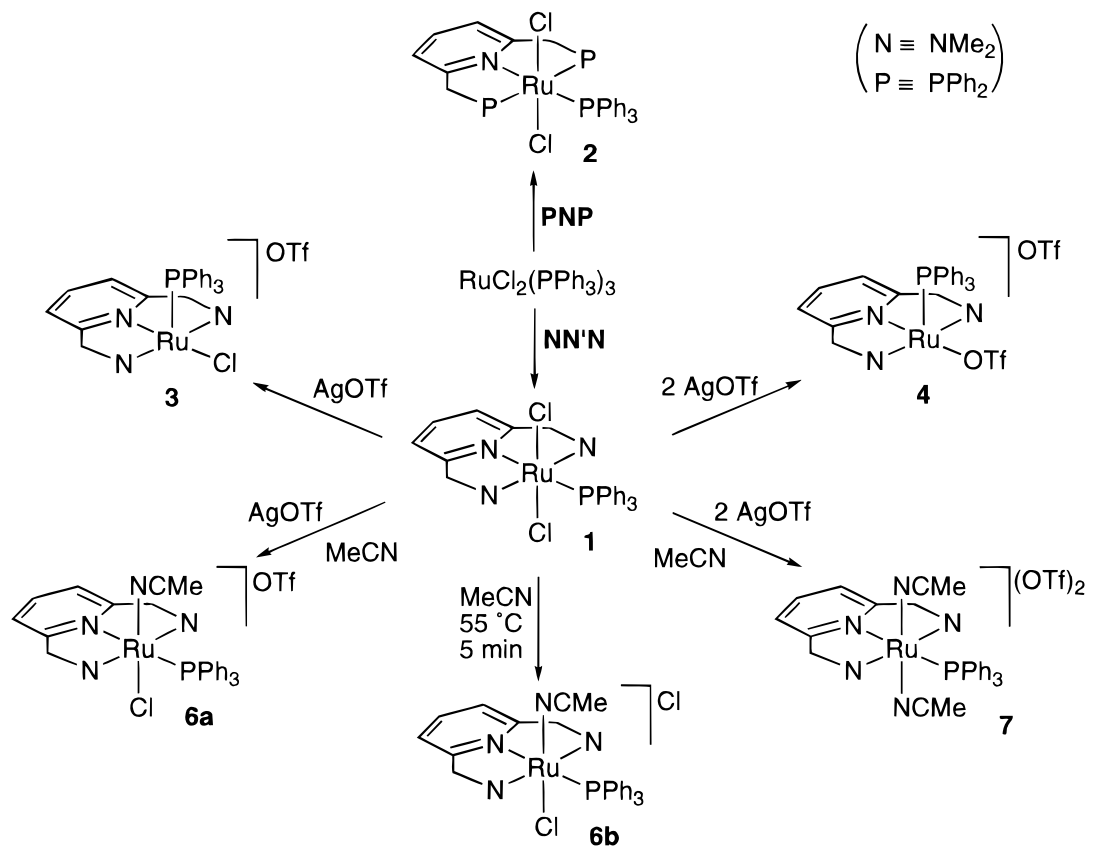

Table 2. Relevant ${ }^{1} \mathrm{H},{ }^{31} \mathrm{P}$, and UV/Vis Data for $\mathbf{N} \mathbf{N}^{\prime} \mathbf{N}-\mathrm{Ru}^{\mathrm{II}}$ Complexes $^{a}$

\begin{tabular}{|c|c|c|c|c|c|}
\hline \multirow[b]{2}{*}{ complex } & \multicolumn{3}{|c|}{${ }^{1} \mathrm{H} \mathrm{NMR}^{a, b}$} & \multirow{2}{*}{$\frac{{ }^{31} \mathrm{P} \mathrm{NMR}^{a, c}}{\mathrm{PPh}_{3}}$} & \multirow{2}{*}{$\begin{array}{l}{\mathrm{UV} / \text { vis }^{d}}^{d} \\
\lambda_{\max }(\epsilon)\end{array}$} \\
\hline & $\mathrm{NMe}_{2}$ & $\mathrm{CH}_{2}{ }^{e}$ & $p$-HNNN $\mathbf{N}^{f}$ & & \\
\hline $\mathbf{N N}^{\prime} \mathbf{N}$ & 2.20 & 3.50 & 7.54 & & \\
\hline 1 & 2.10 & 4.00 & 7.65 & 37.0 & $390(4949)$ \\
\hline $3^{g}$ & $2.68,2.32$ & $4.05,3.51(16)$ & $h$ & 86.6 & 365 (11 144), $530(3500)$ \\
\hline 4 & $2.71,2.31$ & $3.95,3.21(16)$ & 7.75 & 74.5 & $342(5532), 508(1691)$ \\
\hline $6 \mathbf{a}$ & $2.37,2.11$ & $4.30,3.89(15)$ & 7.87 & 32.8 & $350(4663)$ \\
\hline $7^{i}$ & 2.20 & 4.14 & 8.10 & 37.4 & $282(5933)$ \\
\hline
\end{tabular}

${ }^{a} \mathrm{In} \mathrm{CDCl}_{3}$ at $298 \mathrm{~K}$ (unless stated otherwise). ${ }^{b} 300 \mathrm{MHz}$. Chemical shift values ( $\delta$, ppm) indirectly referenced to $\mathrm{SiMe}_{4}$ using solvent signals. ${ }^{c} 81 \mathrm{MHz}$. Chemical shift values $(\delta, \mathrm{ppm})$ are relative to $85 \% \mathrm{H}_{3} \mathrm{PO}_{4}$ with positive values being downfield from the standard. ${ }^{d}$ In $\mathrm{CH}_{2} \mathrm{Cl}_{2}, \lambda_{\max }$ in $\mathrm{nm} ; \epsilon$ in L mol${ }^{-1} \mathrm{~cm}^{-1} .{ }^{e}{ }^{2} J_{\mathrm{HAHB}}(\mathrm{Hz})$ given in parentheses. ${ }^{f}$ Triplet. ${ }^{g}$ Acetone- $d_{6} .{ }^{h}$ Not isolated. ${ }^{i}$ DMSO- $d_{6}$.

Table 3. Relevant ${ }^{13} \mathrm{C}$ NMR Data for $\mathbf{N N} \mathbf{N}-\mathrm{Ru}^{\mathrm{II}} \mathrm{Complexes}^{a}$

\begin{tabular}{|c|c|c|c|c|c|c|c|c|c|}
\hline \multirow[b]{2}{*}{ complex } & \multicolumn{3}{|c|}{$\mathbf{N N}^{\prime} \mathbf{N}$} & \multicolumn{6}{|c|}{$\mathrm{PPh}_{3}$} \\
\hline & $\mathrm{CH}_{2}$ & $\mathrm{NMe}_{2}$ & $\mathrm{C}_{\text {ortho }}$ & $\mathrm{C}_{\text {meta }}$ & $\mathrm{C}_{\text {para }}$ & $\mathrm{C}_{i p s o}{ }^{b}$ & $\mathrm{C}_{\text {ortho }}{ }^{c}$ & $\mathrm{C}_{\text {meta }}{ }^{d}$ & $\mathrm{C}_{\text {para }}$ \\
\hline $\mathbf{N N}^{\prime} \mathbf{N}$ & 65.8 & 45.6 & 158.5 & 121.2 & 136.6 & & & & \\
\hline 1 & 73.7 & 55.6 & 161.7 & 119.3 & 135.0 & $138.9(31.7)$ & $127.5(8.3)$ & $134.4(9.2)$ & 128.3 \\
\hline $3^{e}$ & 73.7 & $52.8,47.6$ & 162.7 & 122.6 & 137.3 & $134.3(51.9)$ & $129.2(10.5)$ & $134.4(9.9)$ & 131.2 \\
\hline 4 & 72.1 & $52.6,45.7$ & 162.6 & 122.6 & 137.8 & $131.6(46.1)$ & $128.9(10.6)$ & $133.4(9.6)$ & 131.3 \\
\hline 6a & 73.6 & $55.1,55.7$ & 159.6 & 121.0 & 137.8 & $136.1(35.5)$ & $128.6(8.8)$ & $133.0(9.6)$ & 129.4 \\
\hline $7^{f}$ & 75.1 & 57.2 & 161.4 & 123.9 & 141.8 & 136.5 (37.9) & $131.6(9.4)$ & $135.0(9.8)$ & 132.5 \\
\hline
\end{tabular}

a $75.469 \mathrm{MHz}, \mathrm{CDCl}_{3}$ at $298 \mathrm{~K}$ (unless stated otherwise). Chemical shift values ( $\delta$, ppm) indirectly referenced to $\mathrm{SiMe}_{4}$ using solvent signals.

${ }^{b}{ }^{1} J_{\mathrm{PC}}$ given in parentheses. ${ }^{c} J(\mathrm{P}, \mathrm{C})$ given in parentheses. ${ }^{d}{ }^{3} J_{\mathrm{PC}}$ given in parentheses. ${ }^{e}$ Acetone- $d_{6} .{ }^{f} \mathrm{DMSO}-d_{6}$.

of $\left[\right.$ mer,trans- $\left.\mathrm{RuCl}_{2}\left(\mathbf{N N}^{\prime} \mathbf{N}\right)\left(\mathrm{PPh}_{3}\right)\right](\mathbf{1})$ only. No mer,cis product is formed according to the ${ }^{31} \mathrm{P}\left\{{ }^{1} \mathrm{H}\right\}$ NMR spectrum. The crystal structure of 1 (Figure 2) also reveals this mer,trans configuration. Some selected crystallographic data for $\mathbf{1}$ are summarized in Table 4.

In contrast to $\mathrm{RuCl}_{2}\left(\mathrm{PPh}_{3}\right)_{3}, \mathrm{RuCl}_{2}\left(\mathrm{PMe}_{3}\right)_{4}$ does not react with the $\mathbf{N N}^{\prime} \mathbf{N}$ ligand at room temperature in $\mathrm{CH}_{2} \mathrm{Cl}_{2}$. Obviously, the $\mathrm{PMe}_{3}$ ligands are too strongly bound to ruthenium(II) for a ligand exchange reaction to take place. Accordingly, $\mathrm{PMe}_{3}$ was found to react with complex 1 with dissociation of both $\mathbf{N N}^{\prime} \mathbf{N}$ and less basic ${ }^{31} \mathrm{PPh}_{3}$ to $\mathrm{RuCl}_{2}\left(\mathrm{PMe}_{3}\right)_{4}$.

Using the same type of ligand exchange reaction of $\mathrm{RuCl}_{2}-$ $\left(\mathrm{PPh}_{3}\right)_{3}$ with PNP, the structurally closely related complex

(31) Basicity of phosphines: ${ }^{32} \mathrm{PPh}_{3}, \mathrm{p} K_{\mathrm{a}}=2.73 ; \mathrm{PMe}_{3}=8.65$

(32) Henderson, W. A., Jr.; Streuli, C. A. J. Am. Chem. Soc. 1960, 82, $5791-5794$
$\left[\mathrm{RuCl}_{2}(\mathbf{P N P})\left(\mathrm{PPh}_{3}\right)\right]$ (2) was obtained. ${ }^{7 \mathrm{~d}}$ Also, the ${ }^{1} \mathrm{H}$ NMR spectrum of $\mathbf{2}$ shows a singlet resonance for the benzylic protons indicating again a meridional coordination mode of the PNP ligand with trans-positioned chloride ligands. Moreover, the ${ }^{31} \mathrm{P}\left\{{ }^{1} \mathrm{H}\right\}$ NMR spectrum of $\mathbf{2}$ shows a set of a doublet and a triplet resonance with a coupling constant of $29 \mathrm{~Hz}$ which is characteristic for a $\mathrm{MX}_{2} \mathrm{Y}$ ligand system and is independent proof for the cis arrangement of $\mathrm{PPh}_{3}$ and the phosphine substituents of the PNP ligand.

Reaction of 1 with Silver Triflate, Preparation of [RuCl$\left.\left(\mathbf{N N}^{\prime} \mathbf{N}\right)\left(\mathbf{P P h}_{3}\right)\right]$ OTf (3) and $\left[\operatorname{RuOTf}\left(\mathbf{N N}^{\prime} \mathbf{N}\right)\left(\mathbf{P P h}_{3}\right)\right]$ OTf (4). When a solution of complex 1 in $\mathrm{CH}_{2} \mathrm{Cl}_{2}$ is reacted with either 1 or 2 equiv of AgOTf, purple complexes $\operatorname{RuCl}(\mathrm{OTf})\left(\mathbf{N N}^{\prime} \mathbf{N}\right)$ $\left(\mathrm{PPh}_{3}\right)$ (3) and $\mathrm{Ru}(\mathrm{OTf})_{2}\left(\mathbf{N N}^{\prime} \mathbf{N}\right)\left(\mathrm{PPh}_{3}\right)$ (4), respectively, are obtained. The ${ }^{19} \mathrm{~F}\left\{{ }^{1} \mathrm{H}\right\}$ NMR spectrum of $\mathbf{4}$ in $\mathrm{CDCl}_{3}$ at 300 $\mathrm{K}$, shows two distinct singlet resonances at -77.82 and -78.17 


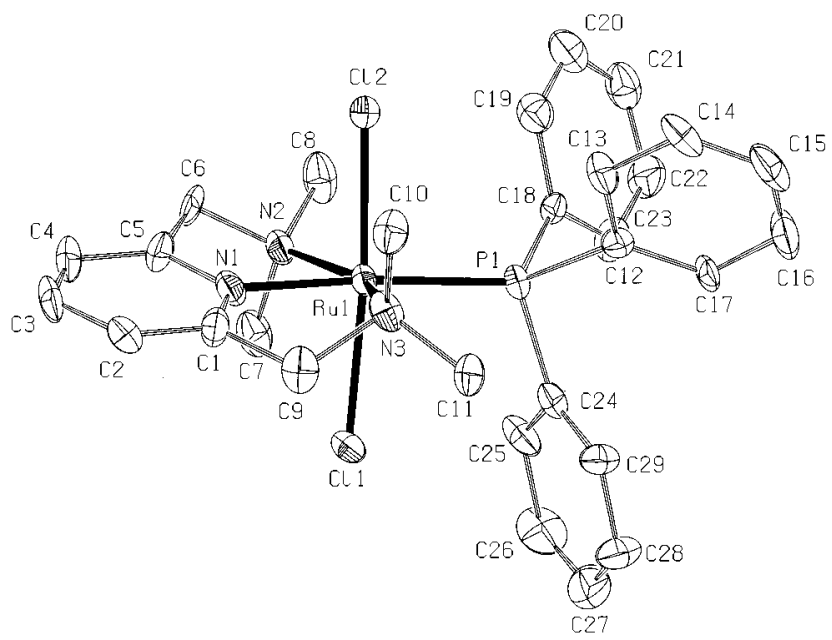

Figure 2. Thermal motion ellipsoid plot (50\% probability level) of $\mathbf{1}$. $\mathrm{H}$ atoms are omitted for clarity.

Chart 1. Potential Coordination Modes of $\mathbf{N N}^{\prime} \mathbf{N}$ in $\mathrm{Ru}^{\mathrm{II}}[\mathbf{N N} \mathbf{N}]$ Complexes

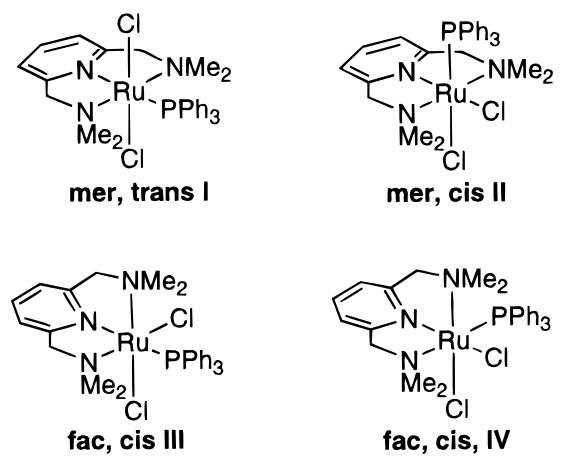

ppm. The signal at $-78.17 \mathrm{ppm}$ has been assigned to a free triflate anion; ${ }^{33}$ this is supported by the observation that the ${ }^{19} \mathrm{~F}$ NMR signal of $\left[\mathrm{Bu}_{4} \mathrm{~N}\right] \mathrm{OTf}$ in $\mathrm{CDCl}_{3}$ under the same conditions occurs at $-78.27 \mathrm{ppm}$. The ${ }^{19} \mathrm{~F}$ NMR spectrum of $\mathrm{RuCl}(\mathrm{OTf})-$ $\left(\mathbf{N N}^{\prime} \mathbf{N}\right)\left(\mathrm{PPh}_{3}\right)$ in acetone- $d_{6}$ shows only one signal for free triflate anion at $-77.76 \mathrm{ppm}\left(\left[\mathrm{Bu}_{4} \mathrm{~N}\right] \mathrm{OTf}:-77.73 \mathrm{ppm}\right)$. These observations lead to the conclusion that these complexes can be best described as cationic 16-electron species $\left[\mathrm{RuCl}\left(\mathbf{N N}^{\prime} \mathbf{N}\right)\right.$ $\left.\left(\mathrm{PPh}_{3}\right)\right] \mathrm{OTf}(\mathbf{3})$ and $\left[\mathrm{RuOTf}\left(\mathbf{N N}^{\prime} \mathbf{N}\right)\left(\mathrm{PPh}_{3}\right)\right] \mathrm{OTf}(\mathbf{4})$ respectively. The ${ }^{1} \mathrm{H}$ NMR spectra of both $\mathbf{3}$ and $\mathbf{4}$ consist of a characteristic $A X$ pattern for the benzylic protons and two distinct resonances for the $\mathrm{NMe}_{2}$ groups, indicating $C_{2 v}$ symmetry in the complexes. Moreover, the ${ }^{31} \mathrm{P}\left\{{ }^{1} \mathrm{H}\right\}$ NMR spectra of both $\mathbf{3}$ and $\mathbf{4}$ show a sharp singlet resonance at relatively low field $(\delta=85.6$ and $74.7 \mathrm{ppm}$, respectively), which is characteristic for a deshielded $\mathrm{PPh}_{3}$ donor. Such low-field resonances of $\mathrm{PPh}_{3}$ have also been observed for the related five-coordinate, neutral 16-electron ruthenium(II) complexes $\left[\mathrm{RuCl}(\mathrm{NCN})\left(\mathrm{PPh}_{3}\right)\right]$ and $[\mathrm{RuI}(\mathrm{NCN})-$ $\left.\left(\mathrm{PPh}_{3}\right)\right](5)\left(\delta=91.1\right.$ and $89.0 \mathrm{ppm}$, respectively $\left.{ }^{10}\right)$ containing the terdentate, monoanionic $\left[\mathrm{C}_{6} \mathrm{H}_{3}\left(\mathrm{CH}_{2} \mathrm{NMe}_{2}\right)_{2}-2,6\right]^{-}\left([\mathrm{NCN}]^{-}\right)$ ligand. The structural description of these complexes in solution is similar to their structure in the solid state.

Suitable crystals of $\mathbf{4}$ were grown from a $\mathrm{CH}_{2} \mathrm{Cl}_{2}$ solution layered with pentane. The solid state structure of $\mathbf{4}$ (Figure 3) clearly shows the separation of cation and anion. Some selected crystal data are summarized in Table 4 . The $\eta^{1}$-bonding mode of the triflate anion is rather unusual in ruthenium coordination

(33) Hollis, T. K.; Robinson, N. P.; Bosnich, B. Organometallics 1992, $11,2745-2748$. chemistry, and only a limited number of ruthenium complexes with such anions have been reported. ${ }^{34}$ The molecular structure of the $\left[\operatorname{RuOTf}\left(\mathbf{N N}^{\prime} \mathbf{N}\right)\left(\mathrm{PPh}_{3}\right)\right]$ cation shows a five-coordinate square-pyramidal ruthenium(II) center with one $\eta^{1}-\mathrm{O}$ bonded triflate anion. The five-coordinate square-pyramidal structure of the cation of $\mathbf{4}$ is distorted to a trigonal bipyramidal one by a $77 \%$ movement along the Berry pseudorotation axis toward the latter. ${ }^{35}$ The cationic nature, as well as the square-pyramidal geometry of the $[\mathbf{4}]^{+}$cation of $\mathbf{4}$ found in the solid state, is also consistent with the solution NMR data. In $[4]^{+}$the $\mathbf{N N}^{\prime} \mathbf{N}$ ligand functions as a terdentate ligand system that occupies three positions in the basal plane of the square pyramid; the $\mathrm{N}(2)-$ $\mathrm{Ru}-\mathrm{N}(3)$ angle is $150.7(2)^{\circ}$. The $\eta^{1}$-bonded triflate occupies the other basal position $\left(\angle \mathrm{N}(1)-\mathrm{Ru}-\mathrm{O}(1)=168.50(20)^{\circ}\right)$, while the $\mathrm{PPh}_{3}$ is coordinating apically $(\angle \mathrm{N}(1)-\mathrm{Ru}-\mathrm{P}=95.50$ $\left.(18)^{\circ}\right)$. Interestingly, the monocation $[4]^{+}$is isostructural with the neutral 16-electron $\mathrm{Ru}-\mathbf{N C N}$ complex $\left[\mathrm{RuI}(\mathbf{N C N})\left(\mathrm{PPh}_{3}\right)\right]$ (5). ${ }^{10}$ However, the ruthenium-phosphine distance in the solid state structure of $\mathbf{5}$ (2.1757(7) $\AA$ ) is lengthened significantly to 2.212(2) $\AA$ in the monocation of $\mathbf{4}$, pointing to a weaker $\mathrm{Ru}-\mathrm{P}$ interaction in $[4]^{+}$. On first sight, this is surprising as one would expect the cationic ruthenium center in $[4]^{+}$to have a higher Lewis acidity than the $\mathrm{Ru}$ in $\mathbf{5}$ due to the presence of the monoanionic (NCN) ligand in the latter. Although it is difficult to evaluate the possible influence of the difference in anionic character of iodine and triflate, these results seem to indicate that the $[\mathbf{N C N}]^{-}$ligand (in 5) is a weaker $\sigma$-donating ligand as compared with $\mathbf{N N}^{\prime} \mathbf{N}$ (in 4). However, when compared to the $\mathrm{Ru}-\mathrm{P}$ bond lengths of 2.337(3) and 2.3451(12) $\AA$ in the sixcoordinate complexes $\mathbf{1}$ and $\mathbf{6 a}$, respectively, the $\mathrm{Ru}-\mathrm{P}$ distance in 4 is considerably shortened $(2.212(2) \AA)$. Obviously, the ruthenium center in the five-coordinate $\mathbf{4}$ has a higher Lewis acidic character as compared to both six-coordinate complexes 1 and 6a. Moreover, the Lewis acidic character of the sixcoordinate complexes $\mathbf{1}$ and $\mathbf{6 a}$ is comparable, despite the cationic character of the latter.

Preparation and Characterization of $[\mathrm{mer}-\mathrm{RuCl}(\mathrm{MeCN})-$ $\left.\left(\mathrm{NN}^{\prime} \mathrm{N}\right)\left(\mathrm{PPh}_{3}\right)\right] \mathrm{OTf}(6)$ and $\left[\right.$ mer,trans $-\mathrm{Ru}(\mathrm{MeCN})_{2}\left(\mathrm{NN}^{\prime} \mathrm{N}\right)-$ $\left(\mathbf{P P h}_{3}\right) \mathbf{H O T f}_{\mathbf{2}} \mathbf{( 7 )}$. The chloride ligands in complex $\mathbf{1}$ can be readily substituted for acetonitrile molecules when a solution of $\mathbf{1}$ in dichloromethane is treated with 1 or 2 equiv of AgOTf in the presence of $\mathrm{MeCN}$. In this way, the complexes with the stoichiometry $\left[\mathrm{RuCl}(\mathrm{OTf})\left(\mathbf{N N}^{\prime} \mathbf{N}\right)(\mathrm{MeCN})\left(\mathrm{PPh}_{3}\right)\right](\mathbf{6 a})$ and $[\mathrm{Ru}-$ $\left.(\mathrm{OTf})_{2}\left(\mathbf{N N}^{\prime} \mathbf{N}\right)(\mathrm{MeCN})_{2}\left(\mathrm{PPh}_{3}\right)\right](\mathbf{7})$ were obtained as light yellow solids. The ${ }^{19} \mathrm{~F}\left\{{ }^{1} \mathrm{H}\right\}$ NMR spectrum of 6 in $\mathrm{CDCl}_{3}$ shows one singlet resonance at $-78.26 \mathrm{ppm}$, corresponding to free triflate anion (vide infra). Moreover, the ${ }^{19} \mathrm{~F}$ NMR spectrum of 7 also shows one singlet resonance for free triflate ion at $-77.15 \mathrm{ppm}$ $\left(\left[\mathrm{Bu}_{4} \mathrm{~N}\right] \mathrm{OTf}:-77.17 \mathrm{ppm}\right)$. These observations point to the formation of mono- and dicationic complexes of type [RuCl$\left.(\mathrm{MeCN})\left(\mathbf{N N}^{\prime} \mathbf{N}\right)\left(\mathrm{PPh}_{3}\right)\right] \mathrm{OTf}(\mathbf{6 a})$ and $\left[\mathrm{Ru}(\mathrm{MeCN})_{2}\left(\mathbf{N N}^{\prime} \mathbf{N}\right)\left(\mathrm{PPh}_{3}\right)\right]-$ $\mathrm{OTf}_{2}(\mathbf{7})$, respectively. The proton NMR spectrum of $\mathbf{6 a}$ (in $\mathrm{CDCl}_{3}$ ) shows an $A X$ resonance pattern for the benzylic protons and two distinct singlet resonances for $\mathrm{NMe}_{2}$ units, indicating that the benzylic carbons and the nitrogen atoms of the $\mathrm{NMe}_{2}$ units lie in a molecular plane of symmetry. In the ${ }^{31} \mathrm{P}\left\{{ }^{1} \mathrm{H}\right\} \mathrm{NMR}$ spectrum, a singlet resonance appears at $32.8 \mathrm{ppm}$ which is illustrative for triphenylphosphine coordinating trans to the pyridine nitrogen.

(34) (a) Blosser, P. W.; Gallucci, J. C.; Wojcicki, A. Inorg. Chem. 1992, 31, 2376. (b) Kraakman, M. J. A.; De Klerk-Engels, B.; De Lange, P. P. M.; Vrieze, K.; Spek, A. L. Organometallics 1992, 11, 3774.

(35) Holmes, R. R. Prog. Inorg. Chem. 1984, 32, 119 and references cited therein. 
Table 4. Selected Bond Distances $(\AA)$ and Angles (deg) for Complexes 1, 4, 6a, 11, and 13

\begin{tabular}{|c|c|c|c|c|c|}
\hline & 1 & 4 & $6 \mathbf{a}$ & 11 & 13 \\
\hline \multicolumn{6}{|c|}{ Bond Distances } \\
\hline $\mathrm{Ru} 1-\mathrm{N} 1$ & $2.023(8)$ & $1.953(7)$ & $2.024(4)$ & $2.058(7)$ & $1.973(2)$ \\
\hline $\mathrm{Ru} 1-\mathrm{N} 2$ & $2.229(10)$ & $2.143(6)$ & $2.226(4)$ & $2.230(7)$ & $2.188(3)$ \\
\hline $\mathrm{Ru} 1-\mathrm{N} 3$ & $2.211(8)$ & $2.151(6)$ & $2.222(4)$ & $2.201(6)$ & $2.177(3)$ \\
\hline $\mathrm{Ru} 1-\mathrm{P} 1$ & $2.337(3)$ & $2.212(2)$ & $2.3451(12)$ & $2.2384(2)$ & - \\
\hline $\mathrm{Ru} 1-\mathrm{Cl1}$ & $2.421(3)$ & - & $2.4060(14)$ & $2.445(2)$ & - \\
\hline $\mathrm{Ru} 1-\mathrm{Cl} 2$ & $2.429(3)$ & - & - & - & - \\
\hline $\mathrm{Ru} 1-\mathrm{OSO}_{2}$ & - & $2.141(6)$ & - & - & $2.143(2)$ \\
\hline Ru1-HOEt & - & - & - & - & $2.159(2)$ \\
\hline Ru1-N4 & - & - & $1.996(4)$ & - & - \\
\hline $\mathrm{Ru} 1-\mathrm{CO}$ & - & - & - & $1.851(8)$ & $1.815(3)$ \\
\hline \multicolumn{6}{|c|}{ Bond Angles } \\
\hline $\mathrm{N} 2-\mathrm{Ru} 1-\mathrm{N} 3$ & $155.8(3)$ & $150.7(2)$ & $156.16(14)$ & 157.1(3) & $160.65(9)$ \\
\hline $\mathrm{N} 1-\mathrm{Ru} 1-\mathrm{N} 2$ & $77.9(3)$ & $81.3(2)$ & $78.00(16)$ & 78.3(3) & $80.19(13)$ \\
\hline $\mathrm{N} 1-\mathrm{Ru} 1-\mathrm{N} 3$ & $78.0(3)$ & $79.7(3)$ & $78.18(16)$ & $78.7(3)$ & $80.55(13)$ \\
\hline $\mathrm{Cl1}-\mathrm{Ru} 1-\mathrm{C} 12$ & $174.48(8)$ & - & - & - & - \\
\hline $\mathrm{Cl} 1-\mathrm{Ru} 1-\mathrm{N} 1$ & $84.7(3)$ & - & $89.53(11)$ & $87.70(19)$ & - \\
\hline $\mathrm{Cl} 1-\mathrm{Ru} 1-\mathrm{N} 2$ & $92.8(3)$ & - & $93.13(10)$ & $93.0(2)$ & - \\
\hline $\mathrm{Cl} 1-\mathrm{Ru} 1-\mathrm{N} 3$ & $86.8(3)$ & - & $87.70(10)$ & $86.32(16)$ & - \\
\hline $\mathrm{Cl1}-\mathrm{Ru} 1-\mathrm{N} 4$ & - & - & $175.89(11)$ & - & - \\
\hline $\mathrm{N} 1-\mathrm{Ru} 1-\mathrm{P} 1$ & $175.9(3)$ & $95.50(18)$ & $177.67(11)$ & $178.97(19)$ & - \\
\hline $\mathrm{N} 2-\mathrm{Ru} 1-\mathrm{P} 1$ & $102.0(2)$ & $103.97(16)$ & $101.91(10)$ & $101.9(2)$ & - \\
\hline N3-Ru1-P1 & $102.1(2)$ & $99.92(18)$ & $101.84(10)$ & 101.1(2) & - \\
\hline $\mathrm{P} 1-\mathrm{Ru} 1-\mathrm{OSO}_{2}$ & - & $94.27(16)$ & - & - & - \\
\hline $\mathrm{N} 1-\mathrm{Ru} 1-\mathrm{OSO}_{2}$ & - & $168.5(2)$ & - & - & $170.07(11)$ \\
\hline $\mathrm{Cl} 1-\mathrm{Ru} 1-\mathrm{CO}$ & - & - & - & $177.3(3)$ & - \\
\hline $\mathrm{EtOH}-\mathrm{Ru} 1-\mathbf{C O}$ & - & - & - & - & $176.78(12)$ \\
\hline $\mathrm{N} 1-\mathrm{Ru} 1-\mathrm{CO}$ & - & - & - & $89.6(3)$ & $94.31(13)$ \\
\hline $\mathrm{N} 2-\mathrm{Ru} 1-\mathrm{CO}$ & - & - & - & $86.7(3)$ & $93.68(13)$ \\
\hline $\mathrm{N} 3-\mathrm{Ru} 1-\mathrm{CO}$ & - & - & - & $92.9(3)$ & $89.48(13)$ \\
\hline
\end{tabular}

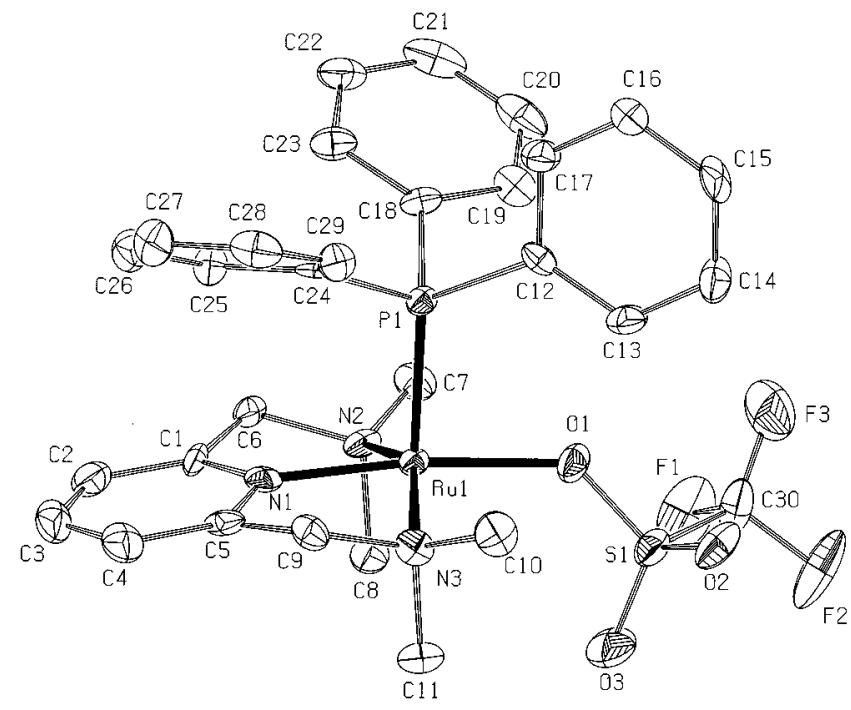

Figure 3. Thermal motion ellipsoid plot (50\% probability level) of 4 . $\mathrm{H}$ atoms are omitted for clarity.

The solid state molecular structure of 6a (Figure 4) shows meridional coordination of $\mathbf{N N}^{\prime} \mathbf{N}$ with the chloride and $\mathrm{MeCN}$ ligand in apical positions and a $\mathrm{PPh}_{3}$ trans-positioned relative to the pyridine nitrogen. Some selected bond distances and angles are given in Table 4.

The NMR data for complex 7 indicate a high degree of symmetry within a complex cation; in its ${ }^{1} \mathrm{H}$ NMR spectrum (DMSO- $d_{6}$ ) the $\mathbf{N N}^{\prime} \mathbf{N}$ ligand shows singlet resonances for the four methylene protons and for the methyl groups of the $\mathrm{NMe}_{2}$ units. It also reveals the presence of two coordinating MeCN ligands. The ${ }^{31} \mathrm{P}\left\{{ }^{1} \mathrm{H}\right\}$ NMR spectrum of complex 7 shows, like those of $\mathbf{1}$ and $\mathbf{6 a}$, a high-field singlet resonance (37.4 ppm). These data suggest a simple trans,mer-I (see Chart 1) octahedral geometry at the dicationic metal, with $\mathbf{N N}^{\prime} \mathbf{N}$ coordinating meridionally.

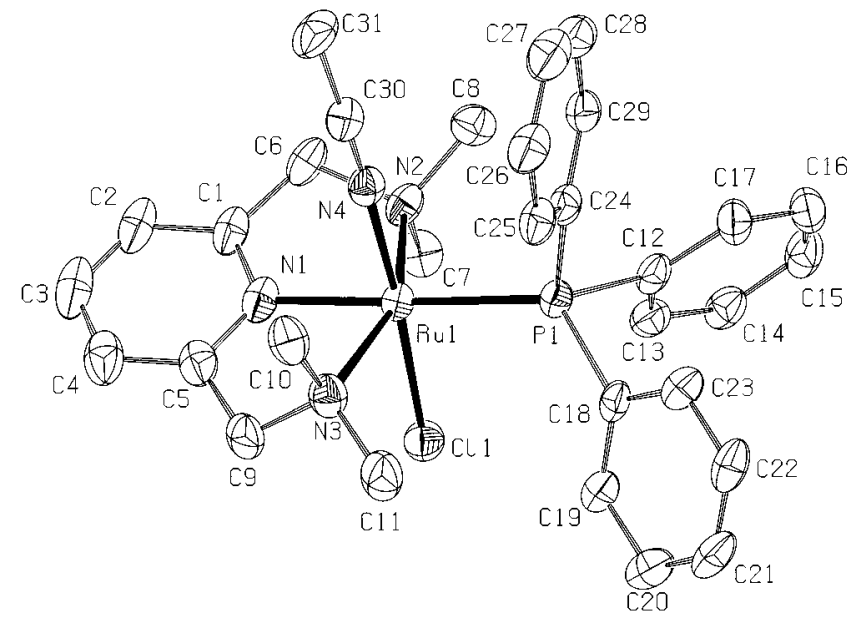

Figure 4. Thermal motion ellipsoid plot (50\% probability level) of 6a. $\mathrm{H}$ atoms are omitted for clarity.

The lability of chloride in complex $\mathbf{1}$ is clearly demonstrated by its reaction with acetonitrile in the absence of silver triflate. When a suspension of $\mathbf{1}$ in acetonitrile is gently heated at 55 ${ }^{\circ} \mathrm{C}$ for a few minutes, a clear brown solution is formed, which, after evaporation of $\mathrm{MeCN}$, yields a yellow-brown solid with molecular formula $\left[\mathrm{RuCl}_{2}(\mathrm{MeCN})\left(\mathbf{N N}^{\prime} \mathbf{N}\right)\left(\mathrm{PPh}_{3}\right)\right](\mathbf{6} \mathbf{b})$ in almost quantitative yield. The ${ }^{13} \mathrm{C}$ and ${ }^{31} \mathrm{P}$ NMR spectra of this new complex closely resemble those of the monocationic complex 6a; however, the ${ }^{1} \mathrm{H}$ NMR spectrum in $\mathrm{CDCl}_{3}$ differs. Although the resonances arising from the pyridine and $\mathrm{PPh}_{3}$ units are quite similar, the singlet resonance of coordinated $\mathrm{MeCN}$ is shifted to higher field (from $1.91 \mathrm{ppm}$ in $\mathbf{6 a}$ to $2.15 \mathrm{ppm}$ in $\mathbf{6 b}$ ). Along with this, the chemical shifts of the doublet resonances of the $A X$ pattern of the benzylic protons are significantly shifted (from 4.30 and $3.88 \mathrm{ppm}$ for $\mathbf{6 a}$ to 4.33 and $4.12 \mathrm{ppm}$ ). Upon exchange of one chloride anion for one triflate anion by reaction of $\mathbf{6 b}$ with 1 equiv of AgOTf, the ${ }^{1} \mathrm{H}$ NMR spectrum of the 
product becomes identical with that of $6 \mathbf{a}$. The UV/visible spectrum of $\mathbf{6 b}$ in dichloromethane closely matches the spectrum of 6a. Both complexes show an absorption maximum at the same wavelength $(350 \mathrm{~nm})$ along with an extinction coefficient of about 4600, proving that both complexes must be isostructural and that $\mathbf{6 b}$ can be described as $\left[\mathrm{RuCl}(\mathrm{MeCN})\left(\mathbf{N N}^{\prime} \mathbf{N}\right)\left(\mathrm{PPh}_{3}\right)\right]$ $\mathrm{Cl}$. We believe that the differences observed in the ${ }^{1} \mathrm{H}$ NMR spectra arise from differences in the anion-cation pair associations in $\mathrm{CDCl}_{3}$ solution. ${ }^{7 \mathrm{~d}}$

During the reaction of $\mathbf{1}$ with acetonitrile at $55{ }^{\circ} \mathrm{C}$, no formation of dicationic $\left[\mathrm{Ru}(\mathrm{MeCN})_{2}\left(\mathbf{N N}^{\prime} \mathbf{N}\right)\left(\mathrm{PPh}_{3}\right)\right]^{2+}$, analogous to $\mathbf{7}$, is observed. At reflux conditions, besides the formation of $\mathbf{6 b}$, partial dissociation of triphenylphosphine from $\mathbf{1}$ is observed along with the formation of unidentified side products. From ${ }^{1} \mathrm{H}$ NMR spectra, crystalline samples of $\mathbf{6 b}$ proved to contain non-coordinating $\mathrm{CH}_{2} \mathrm{Cl}_{2}$. Attempts to obtain a $\mathrm{CH}_{2-}$ $\mathrm{Cl}_{2}$-free sample of $\mathbf{6 b}$ by heating powdered crystals of $\mathbf{6 b} \cdot x \mathrm{CH}_{2}-$ $\mathrm{Cl}_{2}$ in vacuo at $80{ }^{\circ} \mathrm{C}$ overnight failed because of partial dissociation of $\mathrm{MeCN}$ and re-formation of $\mathbf{1}$.

Unexpected Formation of ( $\boldsymbol{\mu}$-Dinitrogen $)\left(\left\{\right.\right.$ mer,trans $-\mathbf{R u C l}_{2}$ $\left.\left(\mathbf{N N}^{\prime} \mathbf{N}\right\}_{2}\right)(\mathbf{8})$. When a slurry of polymeric $\left[\mathrm{RuCl}_{2}(\mathrm{nbd})\right]_{n}(\mathrm{nbd}$ $=2,5$-norbornadiene) is reacted with the $\mathbf{N N}^{\prime} \mathbf{N}$ ligand in refluxing benzene under a dinitrogen atmosphere, a yellowbrown reaction mixture is obtained from which, after workup, light reddish-brown crystals of a new ruthenium complex are obtained which readily turn green upon exposure to air. ${ }^{1} \mathrm{H}$ as well as ${ }^{13} \mathrm{C}$ NMR analyses of this crystalline product showed only singlet resonances for the benzylic protons and $\mathrm{NMe}_{2}$ units, pointing to a meridionally coordinating $\mathbf{N N}^{\prime} \mathbf{N}$ ligand. Assuming the presence of a $\mathrm{RuCl}_{2}$ fragment, it was anticipated that the chloride ligands are trans-positioned as observed in the complexes 1 and 2. According to elemental analysis, $\mathrm{C} / \mathrm{H}$ and $\mathrm{C} / \mathrm{N}$ ratios were consistent with a product with molecular formula $\left[\mathrm{N}_{2} \mathrm{Ru}_{2} \mathrm{Cl}_{4}(\mathbf{N N} \mathbf{N})_{2}\right]$, i.e. of two $\mathrm{RuCl}_{2}\left(\mathbf{N N}^{\prime} \mathbf{N}\right)$ moieties and one molecule of $\mathrm{N}_{2}$. However, the analyzed values of both carbon, hydrogen, and nitrogen were consistently lower than the ones calculated. Transition metal complexes containing dinitrogen as a ligand have been extensively studied ${ }^{36}$ in view of their relevance to nitrogen fixation. ${ }^{37}$ In fact, the dinitrogen metal complex $\left[\left(\mathrm{N}_{2}\right) \mathrm{Ru}\left(\mathrm{NH}_{3}\right)_{5}\right]^{2+}$, Allen and Senoff's ion, ${ }^{38}$ was the first stable transition metal-dinitrogen complex synthesized, and it shows a characteristic $v_{\mathrm{N} \equiv \mathrm{N}}$ vibration. However, in the IR spectrum of the new complex, no stretching vibration $v_{\mathrm{N} \equiv \mathrm{N}}$ is observed, which suggests that this complex has a symmetric dimeric structure with a $\mu^{2}$-bridging dinitrogen molecule. Indeed, in the Raman spectrum a strong signal for a $\mathrm{N}-\mathrm{N}$ stretching vibration at $2099 \mathrm{~cm}^{-1}$ is found, proving the presence of a bridging dinitrogen ligand. Support for this assignment is provided by the vibrational data of the tetracationic complexes $\left[\left(\mathrm{NH}_{3}\right)_{5} \mathrm{Ru}\left(\mathrm{N}_{2}\right) \mathrm{Ru}\left(\mathrm{NH}_{3}\right)_{5}\right]\left[\left(\mathrm{BF}_{4}\right)_{4}\right]\left(2100 \mathrm{~cm}^{-1}\right)$ and $\left[\left(\mathrm{NH}_{3}\right)_{5} \mathrm{Ru}-\right.$ $\left.\left(\mathrm{N}_{2}\right) \mathrm{Ru}\left(\mathrm{H}_{2} \mathrm{O}\right)_{5}\right]\left[\left(\mathrm{BF}_{4}\right)_{4}\right]\left(2080 \mathrm{~cm}^{-1}\right) .{ }^{39} \mathrm{c}, 40$

To obtain conclusive evidence about the nature of the crystalline product, a single crystal X-ray determination was

(36) (a) Hidai, M.; Mizobe, Y. Chem. Rev. 1995, 95, 1115-1133. (b) Leigh, G. J. Acc. Chem. Res. 1992, 25, 177-182.

(37) See for example: Gambarotta, S. J. Organomet. Chem. 1995, 500 $(1-2), 117-126$.

(38) Allen, A. D.; Senoff, C. V. J. Chem. Soc., Chem. Commun. 1965 , $621-622$.

(39) (a) X-ray: Treitel, I. M.; Flood, M. T.; Marsh, R. E.; Gray, H. B. J. Am. Chem. Soc. 1969, 91, 6512-6513. (b) Raman spectroscopy: Chatt, J.; Nikolsky, A. B.; Richards, R. L.; Sanders, J. R. J. Chem. Soc., Chem. Commun. 1969, 154-155. (c) MO description: Ondrechen, M. J.; Ratner, M. A.; Ellis, D. E. J. Am. Chem. Soc. 1981, $103,1656-1659$.

(40) Creutz, C.; Taube, H. Inorg. Chem. 1971, 10, 2664.
Table 5. Selected Bond Distances ( $\AA$ ) and Angles (deg) for Complex 8

\begin{tabular}{llll}
\hline \multicolumn{4}{c}{ Bond Distances } \\
Ru1-N11 & $1.9695(19)$ & Ru2-N21 & $1.969(2)$ \\
Ru1-N12 & $2.166(2)$ & Ru2-N22 & $2.172(2)$ \\
Ru1-N13 & $2.169(2)$ & Ru2-N23 & $2.177(2)$ \\
Ru1-C111 & $2.4121(7)$ & Ru1-Cl21 & $2.4223(7)$ \\
Ru1-C112 & $2.4202(6)$ & Ru2-Cl22 & $2.4233(7)$ \\
Ru1-N14 & $1.9562918)$ & Ru2-N24 & $1.953(2)$ \\
N14-N24 & $1.110(3)$ \\
\multicolumn{5}{c}{ Bond Angles } \\
N11-Ru1-N12 & $80.48(9)$ & N21-Ru2-N22 & $80.37(9)$ \\
N11-Ru1-N14 & $177.07(8)$ & N21-Ru2-N24 & $177.83(8)$ \\
N12-Ru1-N13 & $161.61(9)$ & N22-Ru2-N23 & $160.07(9)$ \\
C111-Ru1-Cl12 & $178.71(2)$ & Cl21-Ru3-Cl22 & $177.07(3)$ \\
Ru1-N14-N24 & $172.75(18)$ & Ru2-N24-N14 & $174.10(18)$
\end{tabular}

performed. Some selected bond distances and angles are collected in Table 5. The solid state structure of the product (Figure 5) shows two $\mathrm{RuCl}_{2}\left(\mathbf{N N}^{\prime} \mathbf{N}\right)$ moieties, which are symmetrically bridged by molecular nitrogen. Both $\mathbf{N N}^{\prime} \mathbf{N}$ ligands are coordinating meridonally, with a resulting transpositioning of the chloride ligands.

On basis of this data, the complex can be formulated as $[(\mu$ $\left.\left.\mathbf{N}_{2}\right)\left\{\text { mer,trans }-\mathrm{RuCl}_{2}\left(\mathbf{N N}^{\prime} \mathbf{N}\right)\right\}_{2}\right]\left(\mathbf{8}\right.$, Scheme 2). The $\mathbf{N N}^{\prime} \mathbf{N}$ ligands are twisted in such a way that the planes defined by $\mathrm{N}(11) \mathrm{N}(14) \mathrm{Cl}(11) \mathrm{Cl}(12)$ and $\mathrm{N}(21) \mathrm{N}(24) \mathrm{Cl}(21) \mathrm{Cl}(22)$ are orientated almost perpendicularly, the actual angle being 89.38$(5)^{\circ}$. Moreover, the $\mathrm{Ru}(1)-\mathrm{N}(14)-\mathrm{N}(24)-\mathrm{Ru}(2)$ bonding unit is slightly bent, as indicated by the dihedral angle of $\mathrm{Ru}(1) \mathrm{N}$ (14) $\mathrm{N}(24) \mathrm{Ru}(2)$ of $6(3)^{\circ}$. The $\mathrm{N}(14)-\mathrm{N}(24)$ distance is 1.110 (3) $\AA\left(c f . \mathrm{N}-\mathrm{N}=1.0977 \AA\right.$ for free $\left.\mathrm{N}_{2}{ }^{41}\right)$; consistent with backdonation of electron density from $\mathrm{Ru}^{\mathrm{II}}$ to $\mathrm{N}_{2} \cdot{ }^{39 \mathrm{c}}$ A comparable lengthening of the $\mathrm{N} \equiv \mathrm{N}$ distance to $1.124 \AA$ was also observed for the closely related tetracationic dimer $\left[\left(\mathrm{NH}_{3}\right)_{5} \mathrm{Ru}\left(\mathrm{N}_{2}\right) \mathrm{Ru}-\right.$ $\left.\left(\mathrm{NH}_{3}\right)_{5}\right]\left[\left(\mathrm{BF}_{4}\right)_{4}\right] .{ }^{39 \mathrm{c}}$ The almost perpendicular twisting of the two $\mathbf{N N}^{\prime} \mathbf{N}$ ligand systems in complex $\mathbf{8}$ can be accounted for by a simple orbital argument. $\mathrm{As} \mathrm{Ru}^{2+}$ is a 6-electron metal ion in an octahedral coordination sphere, the $t_{2 g}$ set of orbitals is filled. Therefore, $\pi$ back-donation of electron density from ruthenium $t_{2 \mathrm{~g}}$ orbitals will occur into two orthogonal sets of empty $\pi^{*}$ antibonding orbitals of the nitrogen. By perpendicular twisting of the $\mathrm{RuCl}_{2}\left(\mathbf{N N} \mathbf{N}^{\prime} \mathbf{N}\right)$ moieties, this back-donation will be optimally effective.

Reactions of 3 and 4 with CO. In view of the expected reactivity of our 16-electron, coordinatively unsaturated complexes $\mathbf{3}$ and $\mathbf{4}$ toward potential substrates, we have carried out some reactions with $\mathrm{CO}$.

When $\mathrm{CO}$ is bubbled through a purple solution of the chloride complex $\left[\mathrm{RuCl}\left(\mathbf{N N}^{\prime} \mathbf{N}\right)\left(\mathrm{PPh}_{3}\right)\right] \mathrm{OTf}$ (3) in $\mathrm{CH}_{2} \mathrm{Cl}_{2}$, the color changes from purple to light brown. Upon addition of pentane, a yellow precipitate, consisting mostly of $\left[\operatorname{RuCl}(\mathrm{CO})_{2}\left(\mathbf{N N}^{\prime} \mathbf{N}\right)\right]-$ OTf (10), is obtained. The presence of two coordinating CO ligands in this complex was clearly established by both ${ }^{13} \mathrm{C}$ NMR and IR spectroscopy. The ${ }^{13} \mathrm{C}$ NMR spectrum of $\mathbf{1 0}$ shows two $\mathrm{CO}$ resonances, and in the $v_{\mathrm{CO}}$ region of the IR spectrum two absorptions are observed, pointing to a complex of type cis- $\mathrm{L}_{4} \mathrm{M}(\mathrm{CO})_{2} \cdot{ }^{42}$ The ${ }^{1} \mathrm{H}$ NMR spectrum of $\mathbf{1 0}$ shows a characteristic $\mathrm{AB}$ pattern for the benzylic protons as well as two distinct resonances for the $\mathrm{NMe}_{2}$ units of the $\mathbf{N N}^{\prime} \mathbf{N}$ ligand, characteristic for meridionally coordinating $\mathbf{N N}^{\prime} \mathbf{N}$ together with a cis-orientation of the two CO ligands.

(41) Handbook of Chemistry and Physics, 76th ed.; Lide, R. D., Ed.; CRC Press: New York, 1995; Section 9, p 20.

(42) Cotton, F. A.; Wilkinson, G. Advanced Inorganic Chemistry, 5th ed.; Wiley: New York, 1988; p 1034 


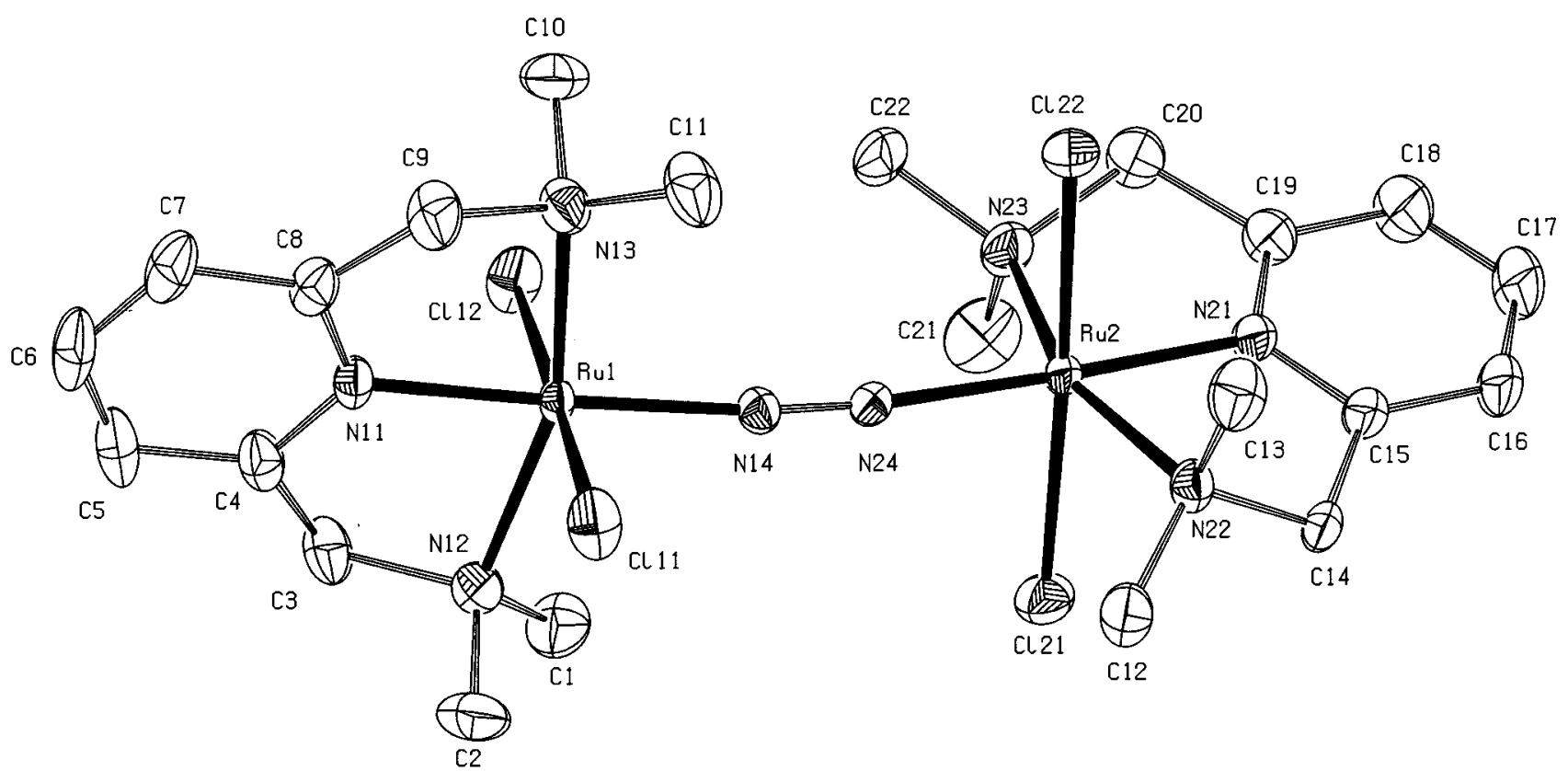

Figure 5. Thermal motion ellipsoid plot (50\% probability level) of $\mathbf{8}$. $\mathrm{H}$ atoms are omitted for clarity.

Scheme 2

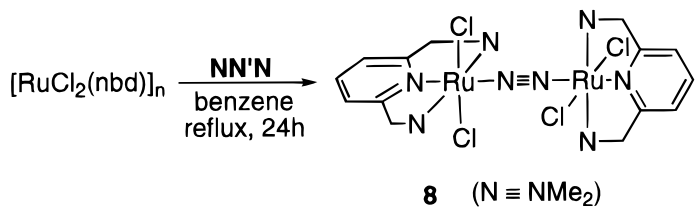

Interestingly, when pentane is allowed to slowly diffuse into the reaction mixture, a compound with molecular formula $\left[\mathrm{RuCl}(\mathrm{CO})\left(\mathbf{N N}^{\prime} \mathbf{N}\right)\left(\mathrm{PPh}_{3}\right)\right] \mathrm{OTf}(\mathbf{1 1})$ crystallizes in high yield. The ${ }^{1} \mathrm{H}$ NMR spectrum of this complex showed diastereotopic resonances of benzylic protons and $\mathrm{NMe}_{2}$ units, indicating a molecular geometry involving meridional terdentate $\mathbf{N N}^{\prime} \mathbf{N}$ coordination. The presence of one coordinating $\mathrm{CO}$ was established by ${ }^{13} \mathrm{C}$ NMR and IR spectroscopy. The ${ }^{13} \mathrm{C}$ NMR spectrum showed only one carbonyl signal, with a small ${ }^{2} J_{\mathrm{PC}}$ coupling constant of $13.6 \mathrm{~Hz}$, pointing to a mutual cis-position of $\mathrm{CO}$ and $\mathrm{PPh}_{3}$. In the $v_{\mathrm{CO}}$ region of the IR spectrum also only one signal was observed. In order to establish the stereochemistry of $\mathbf{1 1}$ and to obtain information about relevant bond angles and distances in the solid state, a single crystal $\mathrm{X}$-ray determination was performed (Figure 6 and Table 4).

The mechanism of formation of $\mathbf{1 0}$ most probably involves the addition of one molecule of $\mathrm{CO}$ to the coordinatively unsaturated 16-electron complex 3 , followed by rearrangement of the intermediate through Berry-pseudorotation intermediates to 11. Subsequent substitution of the $\mathrm{PPh}_{3}$ ligand by $\mathrm{CO}$ then affords 10 (Scheme 3 ). This type of reactivity has also been observed in structurally closely related neutral ruthenium(II) complexes containing the monoanionic ligands $\left[\mathrm{C}_{6} \mathrm{H}_{3}\left(\mathrm{CH}_{2}-\right.\right.$ $\left.\left.\mathrm{NMe}_{2}\right)_{2}-2,6\right]^{-}\left([\mathbf{N C N}]^{-}\right.$and $\left[\mathrm{C}_{6} \mathrm{H}_{3}\left(\mathrm{CH}_{2} \mathrm{PPh}_{2}\right)_{2}-2,6\right]^{-}\left([\mathbf{P C P}]^{-}\right){ }^{10,11}$ For example, reaction of $\left[\mathrm{RuCl}(\mathbf{E C E})\left(\mathrm{PPh}_{3}\right)\right]$ with $\mathrm{CO}$ yields neutral complexes of type $\left[\mathrm{RuCl}(\mathrm{CO})_{2}(\mathbf{E C E})\right]$ with cispositioned $\mathrm{CO}$ ligands, as in 10. The formation of these complexes also proceeds via a neutral monocarbonyl [ $\mathrm{RuCl}$ $\left.(\mathrm{CO})(\mathbf{E C E})\left(\mathrm{PPh}_{3}\right)\right]$ intermediate analogous to $\mathbf{1 0}{ }^{43}$

The reaction of the triflate complex $\left[\operatorname{RuOTf}\left(\mathbf{N N}^{\prime} \mathbf{N}\right)\left(\mathrm{PPh}_{3}\right)\right]$ OTf (4) with $\mathrm{CO}$ was of interest because it could give insight

(43) Karlen, T.; Veldman, N.; Spek, A. L.; van Koten, G. Manuscript in preparation.

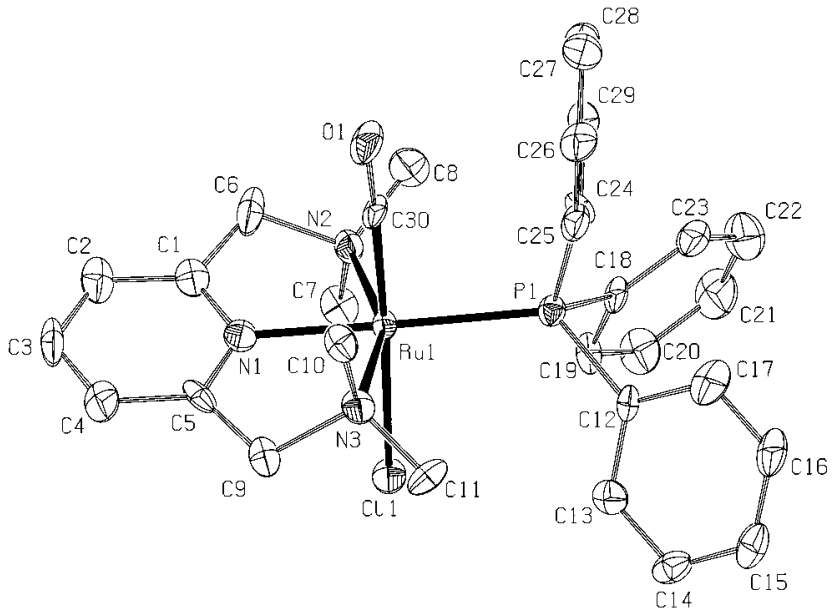

Figure 6. Thermal motion ellipsoid plot (50\% probability level) of 11. $\mathrm{H}$ atoms are omitted for clarity.

Scheme 3

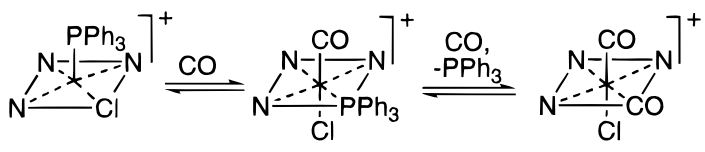

3

11

10

into the nature of the $\mathrm{Ru}-\mathrm{OTf}$ bond. When $\mathrm{CO}$ is bubbled through a purple solution of 4 in $\mathrm{CH}_{2} \mathrm{Cl}_{2}$, a color change from purple to yellow is observed. Independent of the mode of addition of pentane to the reaction mixture, a precipitate is formed existing of at least four unidentified products. The yellow color of these products points to the formation of coordinatively saturated 18-electron complexes. From NMR analyses of this impure yellow solid, it could be concluded that no dissociation of the $\mathbf{N N}^{\prime} \mathbf{N}$ ligand has taken place. The presence of $A X$ resonance patterns in the ${ }^{1} \mathrm{H}$ NMR spectrum points to meridionally coordinating $\mathbf{N N}^{\prime} \mathbf{N}$ in all of the newly formed complexes. One of the products formed (in 10-30\% yield) is the monocarbonyl complex $\left[\right.$ mer-RuOTf $(\mathrm{CO})\left(\mathbf{N N}^{\prime} \mathbf{N}\right)$ $\left.\left(\mathrm{PPh}_{3}\right)\right] \mathrm{OTf}(\mathbf{1 2})$. However, all attempts to isolate a pure product via crystallization failed. Obviously, upon changing from $\mathrm{X}$ 


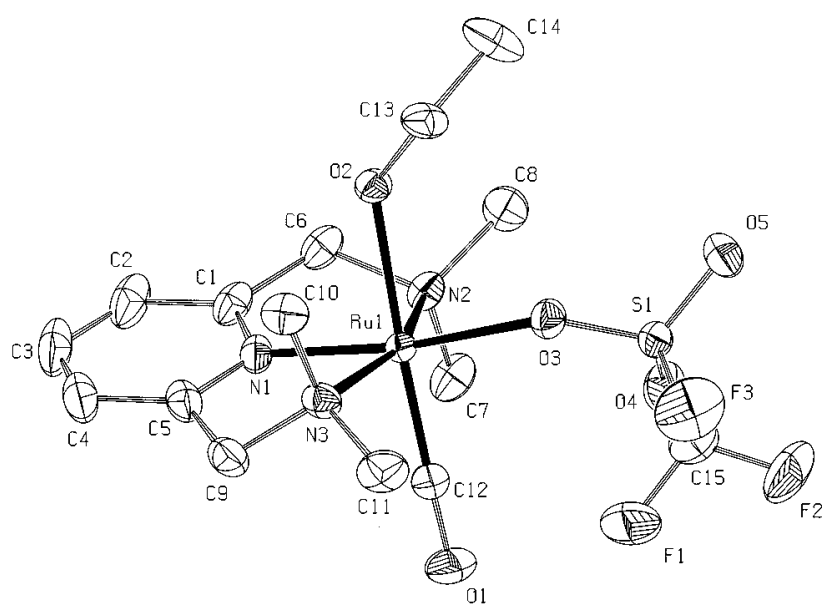

Figure 7. Thermal motion ellipsoid plot (50\% probability level) of 13. $\mathrm{H}$ atoms are omitted for clarity.

Scheme 4

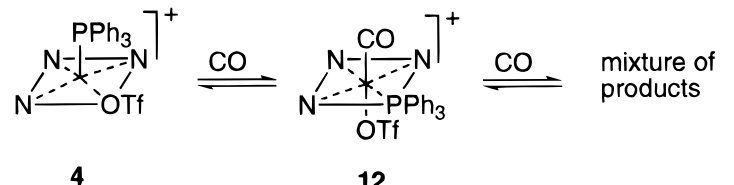

Scheme 5

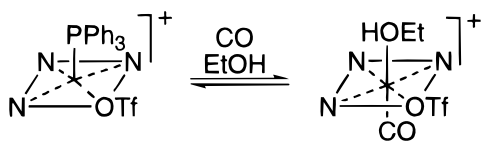

4

13

$=$ chloride in $\mathbf{3}$ to $\mathrm{X}=$ triflate in $\mathbf{4}$, no change of stereoselectivity in the initial reaction with $\mathrm{CO}$ is observed. In both cases, the intermediate monocarbonyl product is formed. However, as stated above, only in the case where $\mathrm{X}=\mathrm{Cl}$ a pure dicarbonyl product could be isolated. It is noteworthy that the structurally related neutral complex $\left[\mathrm{RuOTf}(\mathbf{P C P})\left(\mathrm{PPh}_{3}\right)\right]$ has been reported to be converted into $\left[\right.$ trans $\left.-\mathrm{Ru}(\mathbf{P C P})(\mathrm{CO})_{2}\left(\mathrm{PPh}_{3}\right)\right]$ upon reaction with $\mathrm{CO} .{ }^{43}$

Remarkably, when the same reaction of $\left[\operatorname{RuOTf}\left(\mathbf{N N}^{\prime} \mathbf{N}\right)\right.$ $\left(\mathrm{PPh}_{3}\right)$ ]OTf (4) with $\mathrm{CO}$ is performed in the presence of ethyl alcohol (Scheme 4), upon slow diffusion of pentane in the reaction mixture, large orange to yellow crystals are obtained of which the IR spectrum shows only one resonance in the area of the $v_{\mathrm{CO}}$ absorption frequency, pointing to the presence of only one CO ligand. ${ }^{1} \mathrm{H}$ NMR spectroscopy in acetone- $d_{6}$ or DMSO- $d_{6}$ revealed the presence of one molecule of ethanol in the product. However, due to partial or complete dissociation of the complex in the potentially coordinating solvents used, no NMR data could be obtained. According to elemental analysis, the product can be formulated as $\left[\operatorname{RuOTf}(\mathrm{CO})\left(\mathbf{N N}^{\prime} \mathbf{N}\right)-\right.$ (HOEt)]OTf (13). The solid state structure of $\mathbf{1 3}$ (Figure 7) shows an octahedrally coordinated ruthenium(II) center with meridionally coordinating $\mathbf{N N}^{\prime} \mathbf{N}$. The axial positions are occupied by the ethyl alcohol and the CO ligand. The anionic triflate ligand is trans-positioned toward the central pyridine atom. Some structural data of complex 13 (Scheme 5) are collected in Table 4.

Coordination Behavior of $\mathbf{N N}^{\prime} \mathbf{N}$. In the octahedral 18electron complexes $\mathbf{1},[\mathbf{6}] \mathrm{X}, \mathbf{7}, \mathbf{8}$, and $\mathbf{1 3}$, the $\mathbf{N N}^{\prime} \mathbf{N}$ ligand system is meridionally bonded with the ruthenium center lying in the plane defined by the pyridine ring. The ring strain in the five-membered chelate rings $\mathrm{Ru}-\mathrm{N}(1)-\mathrm{C}-\mathrm{C}-\mathrm{N}(2)$ and $\mathrm{Ru}-\mathrm{N}(1)-\mathrm{C}-\mathrm{C}-\mathrm{N}(3)$ is partially released by ring puckering, which is of $C_{2}$ symmetry, placing one amino methyl group axially on each side of the plane defined by the pyridine ring. The flexibility of the nitrogen donor substituents of the $\mathbf{N N}^{\prime} \mathbf{N}$ ligand is clearly demonstrated in the 16-electron complex $\mathbf{4}$. In this complex, the five-membered chelate rings are puckered with mirror plane symmetry (see plane through $\mathrm{C}(4) \mathrm{NRuO}(1)$ and $\mathrm{P}(1)$ ). Consequently in this structure, one of the methyl groups of each $\mathrm{NMe}_{2}$ unit is axially positioned, and these reside at the same side of the pyridine plane. As a result the remaining $\mathrm{NMe}$ groups are in equatorial position. This structural motif has also been observed in the solid state structure of the related pentacoordinated complex $\left[\mathrm{RuOTf}(\mathrm{NCN})\left(\mathrm{PPh}_{3}\right)\right] .{ }^{10}$ However, in contrast to complexes containing the $[\mathbf{N C N}]^{-}$ligand, ${ }^{10}$ no facial coordination of the $\mathbf{N N}^{\prime} \mathbf{N}$ ligand is found. Although bidentate coordination of terpyridine has been reported, ${ }^{44}$ bidentate coordination of $\mathbf{N N}^{\prime} \mathbf{N}$ has so far not been observed either.

\section{Conclusions}

Although the $\mathbf{N N}^{\prime} \mathbf{N}$ ligand system bears some resemblance to the more rigid terpyridine, complexes containing the $\mathbf{N N}^{\prime} \mathbf{N}$ ligand are not restricted to six-coordination. The $\mathbf{N N}^{\prime} \mathbf{N}$ ligand forms well-defined, five- and six-coordinate $\mathrm{Ru}$ (II) complexes (16- and 18-electron species, respectively) in combination with a variety of unidentate ligands. In all complexes obtained so far, $\mathbf{N N}^{\prime} \mathbf{N}$ uses all three nitrogen donor atoms to coordinate to the metal center in a rather flexible coordination mode. The structural feature of five-coordination results in the formation of coordinatively unsaturated ruthenium(II) complexes containing the $\mathbf{N N}^{\prime} \mathbf{N}$ ligand and makes these complexes interesting from the viewpoint of potential application in homogeneously ruthenium(II)-catalyzed reactions. The presence of a labile dinitrogen ligand makes complex $\mathbf{8}$ potentially interesting as a precursor for the synthesis of a variety of ruthenium(II) complexes of type $\mathrm{RuCl}_{2}\left(\mathbf{N N}^{\prime} \mathbf{N}\right) \mathrm{L}$, where $\mathrm{L}$ is a neutral ligand. We have already found that the dinitrogen can be readily replaced by small molecules like ethylene, ${ }^{45}$ and we are currently investigating this reactivity further.

Acknowledgment. R.A.T.M.A. thanks the Dutch Department of Economic Affairs in terms of the Innovative Oriented Research Program Catalysis (IOP-Katalyse) for financial support. This work was supported in part (A.L.S.) by the Netherlands Foundation for Chemical Research (SON) with financial aid from the Netherlands Organization for Scientific Research (NWO). Mr. W. J. J. Smeets and Mr. D. Kruis are acknowledged for performing X-ray crystallographic studies. I.d.R. thanks the EU for financial support under Grant No. ERBFMBICT961634. Mr. E. T. Lutz is kindly acknowledged for recording Raman spectra.

Supporting Information Available: Two tables of NMR data for the tridentate ligands (1 page). X-ray crystallographic files in CIF format for the structure determinations of complexes 1, 4, 6a, 8, 11, and $\mathbf{1 3}$ are available in the Internet only. Ordering and access information is given on any current masthead page.

\section{IC971061+}

(44) Deacon, G. B.; Patrick, J. M.; Skelton, B. W.; Thomas, N. C.; White, A. H. J. Aust. Chem. 1984, 37, 925.

(45) del Río, I.; Gossage, R. A.; van Koten, G. Eur. J. Inorg. Chem., submitted. 